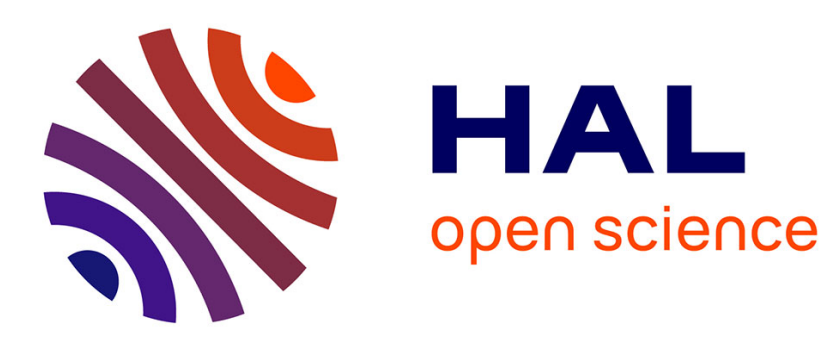

\title{
Comment dater un manuscrit sans le comprendre ? Le cas des archives Condorcet
}

Nicolas Rieucau

\section{To cite this version:}

Nicolas Rieucau. Comment dater un manuscrit sans le comprendre ? Le cas des archives Condorcet. Dix-Huitième Siècle, 2013, 45 (1), 10.3917/dhs.045.0681 . hal-01612935

\section{HAL Id: hal-01612935 \\ https://hal.science/hal-01612935}

Submitted on 11 Oct 2017

HAL is a multi-disciplinary open access archive for the deposit and dissemination of scientific research documents, whether they are published or not. The documents may come from teaching and research institutions in France or abroad, or from public or private research centers.
L'archive ouverte pluridisciplinaire HAL, est destinée au dépôt et à la diffusion de documents scientifiques de niveau recherche, publiés ou non, émanant des établissements d'enseignement et de recherche français ou étrangers, des laboratoires publics ou privés. 


\section{COMMENT DATER UN MANUSCRIT SANS LE COMPRENDRE ? \\ LE CAS DES ARCHIVES CONDORCET}

\section{Nicolas Rieucau}

« D'abord, monsieur, vous avez tort de ne pas dater vos lettres : c'est un avis très important que je vous donne, dussé-je plier le corps en deux pour vous le donner », reproche Julie de Lespinasse au jeune Condorcet ${ }^{1}$. Les lettres de Condorcet à Julie de Lespinasse n'ayant pas été retrouvées, on ne sait s'il observa l'injonction de celle réputée être sa « seconde mère ». Il demeure que, par la suite, les lettres que Condorcet adressera à ses autres correspondants continueront à ne pas être datées de manière systématique : la plupart du temps, on n'y trouve que des mentions cantonnées au quantième du mois (« ce 13 », « ce $22 » \ldots$ ) et/ou aux jours de la semaine (« ce mardi », « ce jeudi »...), voire aucune indication. Le constat de cette négligence, qui semblait aussi partagée par nombre de ses contemporains ${ }^{2}$, peut être étendu à ses textes manuscrits. Si plusieurs centaines d'entre eux sont parvenus jusqu'à nous ${ }^{3}$, extrêmement rares sont ceux datés par Condorcet, si l'on excepte ses rapports et ses certifications de conformité rédigés au titre de l'Académie des sciences, dont la nature officielle exigeait une indication calendaire. Parmi ces pièces rarissimes, deux sont entre elles, par coïncidence, quasi les plus éloignées dans le temps que l'on connaisse. L'une, inédite, est un mémoire adressé à l'Académie des sciences ${ }^{4}$. Il porte l'indication du 23 octobre 1761, alors que Condorcet vient d'avoir 18 ans. L'autre, rédigée quelques mois avant sa mort, est le brouillon de la fameuse Esquisse d'un tableau historique des progrès de l'esprit humain. Y

\footnotetext{
${ }^{1}$ Julie de Lespinasse à Condorcet (13 juil. 1769, EB, p. 29). Afin de lui montrer l'exemple, elle indiquera, en tête de sa lettre suivante, «à Paris, ce 7 août, lundi 1769, neuf heures et demie et 5 minutes du matin et quatre secondes. Temps moyen » (ibid., p. 31).

${ }^{2}$ Par exemple, Rousseau relevait, au sujet de sa propre correspondance : «Diderot ne datoit jamais ses lettres. Made d'Epinay, Made d'Houdetot ne datoit guéres les leurs que du jour de la semaine, et Deleyre faisoient comme elles le plus souvent», Rousseau, Les Confessions (1770, t. I, p. 438). Parmi les mentors de Condorcet, seul Turgot s'attachait à dater ses lettres; d'Alembert et Voltaire n'étant pas des modèles de rigueur en la matière.

${ }^{3}$ Le principal fonds est déposé à la Bibliothèque de l'Institut de France, essentiellement sous les cotes Ms 848 à 885 (III). Un catalogue analytique et matériel est en préparation, dans le cadre de l'établissement de l'Inventaire Condorcet (www.inventaire-condorcet.com).

${ }^{4}$ Essai d'une méthode generale pour integrer les equations differentielles a deux variables, AAS, Pochette de séance du 18 nov. 1761.
} 
figure la date du 4 octobre $1793^{5}$, dont on a du reste de bonnes raisons de penser qu'elle est controuvée ${ }^{6}$.

Afin de dater les lettres et les manuscrits de Condorcet, les considérations relatives au contenu du texte ${ }^{7}$, aux allusions qui peuvent y être faites dans d'autres écrits et aux circonstances historiques et personnelles qui président à sa composition doivent, de façon générale, être souvent combinées à la connaissance des pratiques de rédaction et de la matérialité de l'écrit. Ce sont ces derniers éléments, de nature codicologique ${ }^{8}$, que nous voudrions évoquer dans la présente étude ${ }^{9}$. Ils s'étendent à ceux permettant de reconstituer la genèse de l'œuvre - parce que celle-ci détient une perspective temporelle - lorsqu'on est confronté à plusieurs pièces relevant d'un même projet intellectuel. Ces éléments sont les suivants :

- l'espace graphique et les encres ;

- la forme de l'écriture ;

- les mains ;

- les graphies ;

- le support rédactionnel ;

- les cachets de cire.

La prise en compte de ces caractéristiques matérielles ${ }^{10}$ nous a permis de proposer, de préciser ou de corriger, à ce jour, la datation d'une soixantaine de textes et de plusieurs dizaines de lettres de Condorcet. Nous ne détaillerons pas ici ces résultats, exposés pour la plupart à l'occasion d'autres contributions auxquelles nous nous contenterons de renvoyer au fil de notre étude. En nous attardant en revanche sur les principes nous ayant permis d'obtenir de tels résultats, nous entendons fournir un ensemble d'informations qui, espérons-le, permettront de nourrir les investigations futures d'autres chercheurs s'intéressant aux papiers de Condorcet.

\footnotetext{
${ }^{5} \mathrm{BI}, \mathrm{Ms} 885$ (III), f. $518 \mathrm{r}$.

${ }^{6}$ À ce sujet, voir N. Rieucau (2009, p. 37-38).

${ }^{7}$ Entendu dans un sens large, c'est-à-dire en incluant en particulier les inscriptions allographes portant une date.

${ }^{8}$ Pour une illustration générale de la méthode codicologique appliquée aux manuscrits modernes et centrée sur les instruments et supports d'écriture, voir C. Bustarret (2009).

${ }^{9}$ D'où le titre un peu provocant de celle-ci. La question de la datation des manuscrits de Condorcet n'a fait jusqu'à maintenant l'objet d'aucune recherche si l'on excepte une courte note, rédigée par B. Bru \& P. Crépel (1994, p. 218-220) et que nous avons légèrement complétée dans N. Rieucau (2004 a, p. 177-179).

${ }^{10} \mathrm{Ce}$ qui n'exclut pas qu'il en existe d'autres. Nous pensons notamment au dispositif technique consistant à envisager le feuillet d'un manuscrit comme un image en noir (la trace de l'encre) et blanc (le papier non encré) ; image dont on considère le spectre par des méthodes de transformation de Fourier. Ce procédé permet d'identifier une écriture ou son évolution dans le temps. Pour une présentation, voir L. Hay (1993).
} 


\section{L'espace graphique et les encres}

À l'exception des cas où le texte est très court, les feuillets des premiers jets manuscrits de Condorcet ${ }^{11}$ sont fréquemment divisés en deux colonnes verticales de dimensions similaires, une pliure marquant parfois leur positionnement. Ils sontrédigés la plupart du temps au recto comme au verso ;la colonne située à droite sert d'abord de support rédactionnel, tandis que les révisions un peu étendues et les additions sont inscrites dans la colonne située à gauche, lorsque Condorcet mène une campagne de relecture de son texte ${ }^{12}$. Ce dispositif d'écriture, «à demi-marge $»^{13}$, n'était pas propre à l'académicien. D'origine médiévale, il était encore pratiqué par un grand nombre d'écrivains de son temps et continuera de s'imposer au XIX ${ }^{\mathrm{e}}$ siècle, comme le rappelle C. Bustarret (1996, p. 184). Le texte, une fois mis au net par un copiste ou par Condorcet lui-même, conserve une marge gauche, mais de dimension en général plus réduite. Elle n'occupe plus qu'un tiers, environ, de la largeur du feuillet, cela pour accueillir éventuellement de nouvelles révisions [Document 2, infra, p. 10]. Si celles-ci sont importantes, le manuscrit sera à nouveau mis au net ${ }^{14}$.

Lorsqu'un manuscrit est de la main de Condorcet, l'examen de son espace graphique permet d'emblée de supposer, par un simple regard sur sa marge, qu'il s'agit ou non d'un premier jet. Si la marge est réduite, on peut présumer l'existence d'au moins un autre manuscrit, antérieur, dont Condorcet se serait débarrassé après s'en être servi pour rédiger le manuscrit dont on dispose, à moins que ce manuscrit antérieur n'ait disparu en d'autres circonstances.En outre, si on esten présence deplusieurs manuscrits dont on sait qu'ils relèvent d'un même projet intellectuel, la simple observation de leur espace graphique permet de distinguer les phases rédactionnelles entre elles, d'établir leur stemma et de les situer temporellement, en conséquence, les unes par rapport aux autres. S'il n'est pas possible

\footnotetext{
${ }^{11} \mathrm{Y}$ compris ceux de sa correspondance, quoiqu'il semble rédiger assez rarement des brouillons de ces lettres. Nous n'en connaissons qu'une vingtaine, principalement conservés dans ses papiers aux Archives de l'Académie des sciences et à la Bibliothèque de l'Institut. Une bonne partie de ces brouillons constituent des versions préliminaires de lettres destinées à des sommités. Au sujet des versions finales de ces lettres, voir infra, p. 6 .

${ }_{12}$ Nous exceptons ici les fragments que Condorcet peut ajouter à son texte, dans la mesure où ceux-ci comportent rarement une marge, surtout s'ils sont courts. Néanmoins, puisqu'ils sont très souvent associés à une inscription, dite «de régie», signifiant leur emplacement dans le corps du texte, il est possible d'en déduire qu'ils n'appartiennent précisément pas à une première phase rédactionnelle.

${ }_{13}$ « Il [Condorcet] tenait un papier où il ecrivait à demi-marge les progrès de l'esprit humain », Madame Vernet à Éliza O’Connor (1825 ?, BI, Ms 848, f. 61 r). Rappelons que c'est chez Madame Vernet que Condorcet se réfugiera les derniers mois de sa vie.

${ }^{14}$ Dans leur grande majorité conservés au Ms 856 de la Bibliothèque de l'Institut, les nombreux papiers relatifs à un texte majeur de Condorcet, l'Essai sur les assemblées provinciales (1788), constituent une illustration exemplaire de l'enchaînement de ces diverses phases rédactionnelles.
} 
d'attribuer de ce point de vue une date ou une période précise de rédaction aux diverses pièces auxquelles on est confronté, on peut toutefois déterminer leur filiation dans la genèse de l'œuvre et dresser, de la sorte, une chronologie « interne » aux manuscrits de Condorcet.

À un niveau plus fin, c'est-à-dire sans tenter d'assigner une phase rédactionnelle à telle ou telle pièce, l'analyse de l'espace graphique d'un unique manuscrit révèle la dynamique de son écriture, ce qui permet, là aussi, de la situer dans le temps. Si, au milieu d'un paragraphe, un segment écrit est, par exemple, barré pour être remplacé ou augmenté sur la même ligne d'écriture par un autre segment écrit, on se trouve en présenced'une rature immédiate, intervenue au fil de la plume. En revanche, si la substitution apparaît de façon interlinéaire ou en marge, on a de plus fortes chances d'être confronté à une rature différée, c'est-à-dire opérée lors d'une relecture de plusieurs lignes du paragraphe, de l'intégralité de celui-ci ou d'une partie plus étendue encore du texte.

L'examen de la différence de coloration ou d'épaisseur des encres peut aussi permettre de déceler cette dynamique de l'écriture. Elle doit bien entendu être considérée avec beaucoup de précaution puisqu'elle peut être due à une interruption momentanée, le temps par exemple que Condorcet recharge son encrier ou y trempe à nouveau sa plume. Néanmoins, grâce à cette observation de l'encre, il est parfois possible de repérer l'existence d'une ou de plusieurs campagnes de corrections de l'intégralité d'un manuscrit ou de plusieurs de ses feuillets ${ }^{15}$. L'identification de certaines écritures différées grâce aux variations de l'encre peut, à cet égard, aller jusqu'à permettre de percevoir de véritables réorientations données à un projet intellectuel dans son ensemble ${ }^{16}$.

\section{La forme de l'écriture}

La forme de l'écrituredu jeune Condorcet diffère de celle du Condorcet de la «maturité ». Pour illustrer ce point, figure ci-dessous la reproduction de certains mots dans deux manuscrits. Le premier, que nous avons déjà évoqué, est daté de $1761^{17}$. Le second est rédigé par Condorcet une trentaine d'années plus tard, peu avant sa mort.

\footnotetext{
15 Voir par exemple, s'agissant des Fragments 2, 3, 4 et 9 du Tableau historique de 1793-1794, N. Rieucau (2004 b, p. 1178, 1183-1184, 1187, 1190).

${ }^{16}$ L'exemple le plus fameux est celui de la genèse du Tableau historique de 1793-1794. Condorcet a d'abord envisagé de rédiger une version étendue de celui-ci, avant de se raviser et d'ajouter d'une encre plus foncée « Prospectus d'un » à côté du titre initial « Tableau historique des progrès de l'esprit humain ». À ce sujet, voir P. Crépel (1996, p. 481-484), N. Rieucau (2003, p. 23-25), (2004 b, p. 1161, 1165-1167).

17 Pour autant que nous le sachions, ce manuscrit inédit constitue la plus ancienne trace de l'écriture de Condorcet, si l'on excepte sa signature à l'âge de cinq ans, au bas d'un extrait d'un registre paroissial (10 août 1749), AD Aisne, 5 MI 1520.
} 


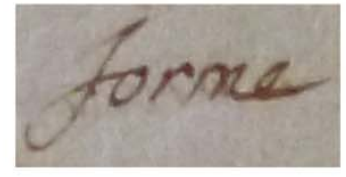

1761

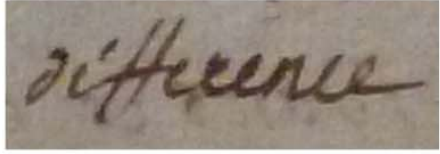

1761

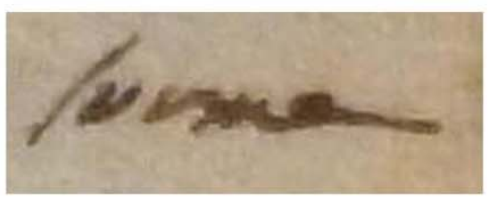

1793-1794

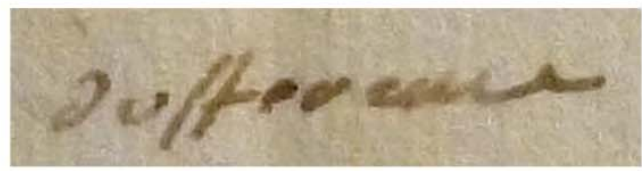

1793-1794

Document 1 : Écriture de certains mots par Condorcet dans l'Essai d'une méthode generale pour integrer les equationsdifferentiellesa deux variables(23 oct. 1761), AAS, Pochette de séance du 18 nov. 1761 et dans l'Exemple des méthodes tecniques(1793-1794), BI, Ms 885 (I), f.125 r, 128 r.

On constate qu'avec le temps, l'écriture de Condorcet a tendance à devenir moins soignée, les mots étant tracés de façon tout à la fois plus ramassée et allongée ${ }^{18}$. Cette analyse de la forme de l'écriture doit cependant être considérée avec beaucoup de précautions, cela pour quatre raisons.

Premièrement, parce qu'elle peut dépendre de la phase rédactionnelle dont relève le manuscrit où elle se trouve. Ainsi, la forme de l'écriture sur un premier jet manuscrit de Condorcet est en général moins appliquée que celle d'une mise au net que ce dernier destine à un copiste. Dans l'exemple exposé ci-dessus, nous avons donc veillé à ce que les deux manuscrits sollicités soient associés à la même phase rédactionnelle - il s'agissait, en l'occurrence, de mises au net.

Deuxièmement, la forme de l'écriture dépend aussi du type de texte rédigé par Condorcet. Par exemple, celle des notes cursives qu'il consigne en tant que Secrétaire perpétuel lors des séances de l'Académie royale des sciences a de fortes chances de différer de celle d'un manuscrit écrit à la même époque dans le calme de son cabinet. La forme de l'écriture d'un manuscrit peut,en tant que telle,ne pas ressembler exactement à celle d'une lettre, parce que Condorcet prend garde dans ses relations épistolaires à ce que ses destinataires ne rencontrent pas de difficultés de lecture, quoique son écriture demeure naturellement assez lisible ${ }^{19}$ et n'a

\footnotetext{
18 On mentionnera aussi une évolution dans le tracé des accents circonflexes. Ceux du jeune Condorcet s'apparentent souvent à des trémas ou à des signes du type «<», ce qui est nettement moins le cas chez le Condorcet de la maturité qui, de manière générale, accentue moins.

${ }^{19}$ Que le texte soit écrit pour lui-même, une institution ou un correspondant. On notera par ailleurs que Condorcet use fort peu d'abréviations, « c' a d » pour « c'est-à-dire » étant presque la seule.
} 
rien à voir avec celle d'unMalesherbes. Il convient en conséquence lors d'une comparaison des formes de l'écriture de s'assurer que celle-ci fasse intervenir des textes de même type.

Troisièmement, dans le cas particulier de la correspondance, la forme de l'écriture de Condorcet peut également dépendre de la personne à qui il s'adresse. On devine en effet que s'il écrit à un proche, qui plus est a priori habitué à le lire, Condorcet fera montre de moins d'application que s'il écrit par exemple à une tête couronnée. Dans le second cas, cette application se manifestera en outre par la rédaction préalable d'un voire de plusieurs brouillons. La lettre originale sera donc une mise au net, dont l'écriture sera d'autant plus aisément déchiffrable ${ }^{20}$. La forme de l'écriture de Condorcet peut, plus prosaïquement, être aussi conditionnée par l'état de santé oculaire de celui qui le lit, en supposant bien sûr que l'académicien en ait connaissance. «Je vous écris de manière pourtant a ne pas fatiguer votre vue » $(\mathrm{CH}, \mathrm{p}$. 196) prévient-il ainsi Turgot, l'un de ses principaux correspondants, dans une lettre vraisemblablement rédigée au début du mois de mars 1775. De fait, l'original de cette lettre $^{21}$ est particulièrement lisible parce que les mots y sont bien écrits et de large taille, se distinguant en cela de ceux que l'on trouve dans d'autres lettres de Condorcet à son mentor, notamment celles du début des années 1770. Mais ces mêmes raisons peuvent évidement intervenir s'il s'adresse à d'autres correspondants sans qu'on le sache, ce qui peut en conséquence introduire un biais dans la datation.

Quatrièmement, le ductus de Condorcet, entendu comme le tracé des lettres dépendant de son état psychique ou physique à un moment donné, peut influencer la forme de son écriture. $\mathrm{Si}$, par exemple, le jeune Condorcet se saisit de la plume dans un accès de colère ${ }^{22}$, son ductus risque de n'être ni relâché ni appliqué, ce qui peut suggérerune datation exagérément tardive de son manuscrit, puisque le tracé de ses lettres s'apparentera alors à celui de la «maturité ». En retour, l'examen du ductus peut permettre de deviner que Condorcet réagit subitement, pour ainsi dire «à chaud », à un évènement qui le touche, en prise directe avec l'actualité de

\footnotetext{
${ }^{20}$ Voir par exemple les deux lettres originales de Condorcet à Frédéric II (mai 1784 et 10 juil. 1786), conservées respectivement aux Archives d'État de Berlin, Rep. 96, Nr. 204 B, et au Musée des lettres et manuscrits de Paris, pièce $\mathrm{n}^{\circ} 37883$ [=5], ou encore la lettre originale adressée à Stanislas II (17 avr. 1791 ?), se trouvant à la Biblioteka Jagiellonska de Cracovie, Autographen Sammlung, Dig. ORP 000492. Non seulement l'écriture de ces trois pièces est particulièrement soignée mais, de plus, les ratures en sont quasi absentes : seule une amorce de mot est barrée sur la lettre à Stanislas II tandis que quelques mots y ont été effacés par grattage pour être substitués avec minutie par d'autres, remplacement méticuleux que l'on trouve aussi à un endroit de la première des deux lettres à Frédéric II précédemment citée. L'espace graphique de ces trois lettres, avec l'adresse " Sire » à laquelle succède un large espace vierge, est également remarquable.

${ }^{21}$ Cet original (numéroté « 68/35») est détenu par un collectionneur. Nous avons pu le consulter, de même que celui de 114 autres lettres de Condorcet à Turgot, grâce au libraire Jean-Baptiste de Proyart. Que ce dernier, ainsi que leur acquéreur, en soient vivement remerciés.

${ }_{22}$ De ce point de vue, l'entreprise de textualisation manuscrite peut avoir chez Condorcet pour finalité l'apaisement de son irritation, sans qu'un passage chez l'imprimeur soit ensuite nécessairement exigé. Il s'agit là d'une des raisons de la présence d'inédits dans ses papiers.
} 
son temps. Un indice de datation peut dans ce cas être décelé ; il peut du reste être renforcé par le constat d'omission de plusieurs mots : emporté par sa fougue, celui que certains de ses proches - J. de Lespinasse, d'Alembert, A. Suard et Turgot - avaient surnommé le « volcan couvert de neige », ou encore le « mouton enragé », pense alors plus vite qu'il ne peut écrire.

La forme de l'écriture de Condorcet ne dépend donc pas uniquement de son âge mais d'un ensemble de paramètres qu'il n'est pas toujours facile de maîtriser. Si on la considère avec quelque réserve et ménagement, son examen demeure néanmoins utile en première approximation lors d'une entreprise de datation, et bien évidemment indispensable lorsqu'on est confronté à un fragment de quelques lignes voire de quelques mots, où aucun autre indice chronologique n'a pu être décelé.

\section{Les mains}

Des années durant, Condorcet n'a pas eu de copiste privilégié, c'est-à-dire de « secrétaire » à proprement parler, comme a pu l'être par exemple Wagnière pour Voltaire ou Garçon pour Mirabeau père. Bien qu'écrite de Brest, et par conséquent hors de son lieu de résidence parisien, l'extrait suivant d'une lettre qu'il adresse à A. Suard au début du mois d'août 1785 suggère cette absence de secrétaire attitré : «Dans ce moment,[...] je vous écris dans un cabaret, pendant que l'on copie un long mémoire que j'ai été obligé de barbouiller » (p. 223). Cet emploi de divers copistes à différents moments de la vie de Condorcet peut servir de guide en matière de datation de ses manuscrits ${ }^{23}$.

Nous sommes à ce jour parvenus à distinguer une vingtaine de copistes différents dans les papiers de Condorcet ${ }^{24}$. Cependant, une étude complète des copistes qu'il a employés tout

\footnotetext{
${ }^{23}$ Pour une illustration, relative au Tableau historique des progrès de l'esprit humain, voir N. Rieucau (2003, p. 19-20). La datation des lettres de Condorcet d'après la reconnaissance de leur main, lorsqu'il ne s'agit pas de celle de l'académicien, est a priori nettement moins fructueuse. Nous n'avons en effet jamais rencontré encore ce cas de figure : sur les presque 600 lettres originales de Condorcet à ce jour répertoriées, la petite dizaine qui ne sont pas de sa main et seulement signées ou brièvement annotées par lui sont systématiquement datées. Comme il le reconnaît dans sa correspondance, Condorcet ne faisait pas non plus exécuter, ni n'exécutait luimême, de minutes de ses lettres. Voir Condorcet à A. Suard (fin juil.-début août 1772, EB, p. 87) et Condorcet à Turgot (1777 ?, CH, p. 296).

${ }^{24}$ Louis Fromage de Longueville (1732 ?-1786) doit jusqu'à preuve du contraire en être exclu. Dans l'étude qu'il lui consacre, A. L'Eleu (1913, p. 183) juge " point déraisonnable », sans avancer d'argument, de considérer qu'il fut employé comme "secrétaire de Condorcet», fonction qui lui est aussi accordée dans l'inventaire des manuscrits de la Bibliothèque de Tonnerre (CGM, $t$. VI, p. 122). Il n'empêche que l'écriture de Fromage de Longueville ne figure pas, sauf erreur de notre part, dans les papiers de Condorcet. Au sujet de ce personnage sulfureux, originaire de Picardie comme Condorcet et qui bénéficia de son aide au milieu des années 1770, on peut consulter, outre l'étude d'A. L'Eleu, celles de B. Vinot (1989 a, p. 418 et surtout 1989 b, p. 7) et de F. Moureau (1999).
} 
au long de sa carrière intellectuelle reste à conduire ${ }^{25}$. Nous nous contenterons d'évoquer ici brièvement cinq d'entre eux, parmi les plus significatifs.

Mérite d'abord d'être signalé un certain «M. Hebert», le premier copiste quisemble avoir été quelque temps au service de Condorcet. On trouve par exemple son écriture dans le manuscrit du Petit ouvrage "plus métaphysique que mathématique » sur le calcul des probabilités (1773-1774) ${ }^{26}$ et dans celui des Réflexions sur les corvées $(1775)^{27}$.

Plus familier est le nom d'Auguste de Keralio (1715-1805), d'abord militaire avant de devenir sous-gouverneur de l'Infant de Parme entre 1757 et $1769^{28}$. Keralio fut spécialement chargé de mettre au net les écrits mathématiques de Condorcet à partir de la seconde moitié des années 1770. Son écriture intervient par exemple sur le manuscrit des deux premières parties (1778-1782) du Traité du calcul intégral ${ }^{29}$, de l'Essai sur l'application de l'analyse à la probabilité des décisions rendues à la pluralité des voix $(1785)^{30}$ ou encore desquatrièmes, cinquièmes et sixièmes parties (1786-1787) du Mémoire sur le calcul des probabilités ${ }^{31}$.

Un autre copiste, dont nous ne sommes pas parvenus à découvrir l'identité, doit être aussi mentionné. Sa particularité est d'avoir assisté Condorcet dans l'exercice de ses tâches de Secrétaire perpétuel de l'Académie royale des sciencesmais également dans la rédaction de certaines lettres ou manuscrits personnels. Sauf erreur de notre part, dans le Plumitif des séances de l'Académie des sciences, les premières et dernières traces de sa main portent respectivement les dates du 21 avril 1784 et du 23 juin 1787. On trouve par ailleurs son écriture dans une lettre de Condorcet à Blagden, rédigée vers mai-juin 1784². Elle figure aussi dans une lettre à Saluces, datée du 19 août de la même année ${ }^{33}$, puis dans divers manuscrits, dont celui de l'Éloge d'Euler $(1785)^{34}$, de De l'influence de la Révolution d'Amérique sur l'Europe $(1786)^{35}$ et des Élemens du calcul des probabilités (1786-1787) ${ }^{36}$.

\footnotetext{
${ }^{25}$ À ce propos, l'analyse systématique du papier utilisé par les différents copistes de Condorcet, qui pourrait être riche d'indications, n'a en particulier pas encore été menée.

${ }^{26} \mathrm{BI}$, Ms 875, f. 100-109. Condorcet nomme explicitement ce copiste dans un passage de son brouillon autographe (BI, Ms 875, f. 87 r). On dispose aussi d'un billet qu'il adresse à Hebert, vraisemblablement en 1774 , à propos de l'acheminement de bonnes feuilles et d'épreuves de divers textes académiques. Nous remercions Jean-Daniel Candaux de nous avoir communiqué ce document issu de sa collection.

${ }^{27}$ BI, Ms 857, f. 216-227 et 233-242.

${ }^{28}$ Au sujet de Keralio, consulter É. Badinter (2008).

${ }^{29} \mathrm{BI}, \mathrm{Ms}$ 879, f. 1-77 (Première partie) et 78-322 (Deuxième partie).

${ }^{30}$ BOP, Z 29, f. 8-13.

${ }^{31}$ BOP, Z 29, f. 2-6, 14-19 ; BI, Ms 854, f. 401-402.

${ }^{32}$ Londres, Royal Society, CB/ 1/ 3/ D15.

${ }^{33}$ Turin, Accademia delle scienze, carton 32225.

${ }^{34} \mathrm{BnF}$, NAF 27261, f. 3 r-10 r, 19 v-33 r.

${ }^{35}$ BI, Ms 857, f. 530-534, 547-552.

${ }^{36} \mathrm{BI}, \mathrm{Ms} 882$, f. 154-159, 162-163.
} 
À ce copiste succèdera Étienne Cardot, dont on peut considérer qu'il fut le premier et le seul véritable «secrétaire» de Condorcet ${ }^{37}$. À l'occasion d'une lettre adressée au Comité de Sûreté Généralele 13 thermidor an II (31 juillet 1794) ${ }^{38}$,Cardotdéclarera être entré au service de Condorcet en juillet 1787 essentiellement pour rédiger «les Ecritures et Copies pour lui » - dans le Plumitif des séances de l'Académie des sciences, sa main apparaît d'ailleurs pour la première fois le 4 juillet 1787, soit deux semaines après la dernière intervention du copiste que nous avons précédemment évoqué ${ }^{39}$. L'écriture de Cardotest reconnaissable, à partir de cette date,dans une multitude de documents d'archives liés à l'activité de l'Académie des sciences, mais aussi dans la grande majorité des copies manuscrites des textes personnels de Condorcet ${ }^{40}$. Cela n'exclut pas le fait que Condorcet ait eu recours aux services de Cardot avant 1787, mais de manière ponctuelle. Depuis le milieu des années 1770, en effet, Cardot vivait sous le même toit que Condorcet, en tant que domestique de son oncle, Claude-Nicolas Gaudry et, comme l'indique A.-M.Chouillet (1997, p. 16), il « était peut-être déjà employé pour des copies personnelles », ce qui « était souvent le cas des domestiques ayant une belle écriture ».

\footnotetext{
${ }^{37}$ À propos de Cardot, voir A.-M. Laffitte-Larnaudie (1997).

${ }^{38}$ AN, F 7 4633, dos. 3.

${ }^{39}$ Les comptes rendus des séances des 27 et 30 juin 1787 sont quant à eux intégralement de la main de Condorcet.

${ }^{40}$ Tant et si bien que l'on compte plusieurs dizaines de pièces de la main de Cardot conservées parmi les papiers de Condorcet à la Bibliothèque de l'Institut (Ms 848-885 (III)).
} 


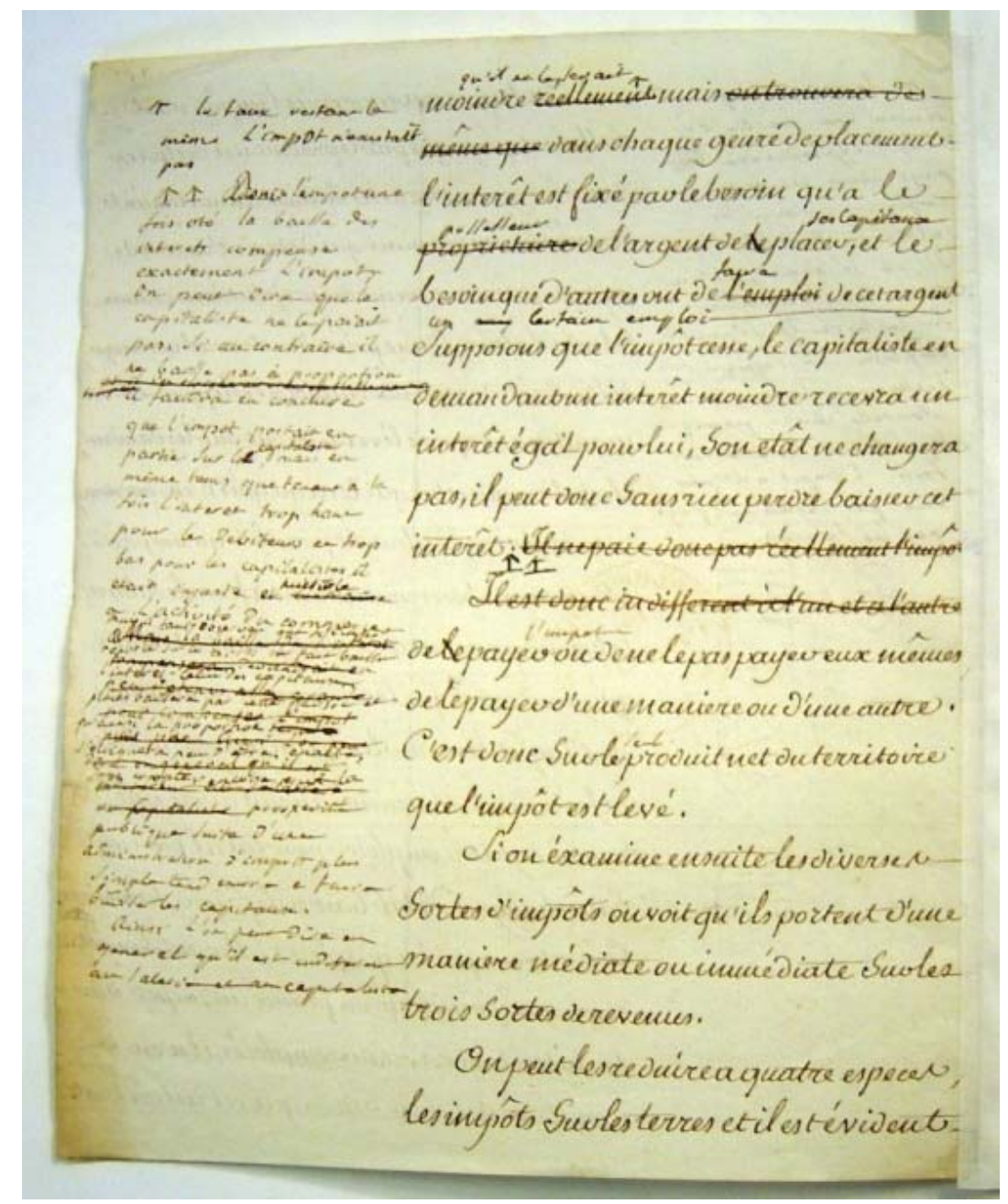

Document 2 : Essai sur les assemblées provinciales (1788), BI, Ms 856, f. 182 v (copie de Cardot corrigée par Condorcet).

Si Cardot devint à partir de l'été 1787 un véritable secrétaire pour Condorcet, il n'empêche que l'académicien continuera d'avoir recours à d'autres plumes pour copier certains de ses manuscrits. Intervient à cet égard assez souvent l'écriture du copiste employé pour mettre au propre, avec Cardot, les Mémoires sur l'instruction publique $(1791)^{41}$. On retrouve son écriture dans des manuscrits tels que le Rapport sur le choix d'une unité de mesure $(1791)^{42}$, la Dernière réflexion sur la procédure écrite ou parlée $(1791)^{43}$, la Révision des travaux de la première législature $(1792)^{44}$ ou encore Sur l'affaire du juge de paix Larivière $(1792)^{45}$.Ce dernier texte, rédigé plus précisément à la fin du printemps ou durant l'été 1792, semble marquer le termed'une collaboration qui aura donc duré près de deux ans.

\footnotetext{
${ }^{41}$ BI, Ms 872, passim ; Ms 884, f. 300-302, 304.

42 BI, Ms 872, f. 591-593, 601-602.

${ }^{43}$ BI, Ms 857, f. 13.

${ }^{44}$ BI, MS 863, f. 204-205.

${ }^{45}$ BI, Ms 863, f. 289-292.
} 


\section{Les graphies}

On sait que les règles orthographiques, qu'elles concernent les noms communs ou les noms propres, ne sont pas encore bien fixées du temps de Condorcet. Si bien qu'au sein d'un même manuscrit, un mot peut revêtir plusieurs graphies différentes. Cela étant, au cours du temps, on relève deux changements particuliers dans les habitudes orthographiques de Condorcet, relatifs respectivement à la terminaison des formes verbales qu'il emploie - par exemple « j'avais » et non « j'avois » - et au doublement de la consonne « $\mathrm{m}$ », perceptible dans le passage de la graphie « home » à la graphie « homme » et qui paraît devoir être étendu à d'autres mots - de « come » à « comme », de « some » à « somme » ${ }^{46} \ldots$

On a tout lieu de penser que le premier de ces changements obéit à une recommandation de Voltaire, comme l'ont remarqué B. Bru \& P. Crépel (1994, p. 218-219). Voltaire militait en effet pour une réforme de l'orthographe française ${ }^{47}$ et notamment pour le passage du « oi » au « ai $»^{48}$. Parmi les proches de Condorcet, des sommités telles que Turgot et d'Alembert ne suivirent pas ses conseils, à la différence semble-t-il de Condorcet, plus jeune et moins célèbre à l'époque. En compagnie de d'Alembert, il visita Voltaire à Ferney en septembre 1770, voyage dont il revint en novembre de la même année, enchanté et ébloui. Par conséquent, c'est fort probablement sous l'influence de Voltaire que Condorcet changea la terminaison de ses formes verbales. Les manuscrits en « oi » doivent donc être considérés comme antérieurs au début de l'année 1771. Bien évidemment, le passage du « oi » au « ai » ne s'est pas réalisé de façon radicale, les habitudes rédactionnelles de Condorcet, si l'on peut dire impulsives, surpassant parfois la règle orthographique qu'il s'était consciemment imposée. C'est pourquoi les manuscrits en « ai » où subsistent quelques occurrences en « oi » ont dû être rédigés après le début de l'année 1771 mais quasi certainement avant le milieu des années 1770. Un manuscrit comportant en revanche un grand nombre d'occurrences en «ai » et aucune en « oi » datera, presque à coup sûr, d'une période postérieure au milieu des années 1770 .

\footnotetext{
${ }^{46} \mathrm{D}$ 'autres évolutions pourraient être approfondies. Par exemple, il semble que la graphie du mot « moien » et de ses dérivés s'efface progressivement chez Condorcet, à partir du milieu des années 1780, au profit de celle de « moyen ». Plus généralement, le « $\mathrm{i}$ » tendrait à être remplacé, dans de nombreuses graphies, par le « $\mathrm{y}$ » dans le yod intervocalique. Indépendamment des questions de datation, signalons au passage que la prise en compte des graphies peut aussi permettre de rapprocher entre eux certains manuscrits isolés ou de remarquer que la connaissance par Condorcet de tel ou tel auteur, parce qu'il en écrit le nom d'une façon que l'on considère fautive, relève de sa culture orale.

${ }^{47}$ Entreprise de façon systématique à l'occasion de la première édition (1751) de l'Essai sur les mœurs. Pour une analyse détaillée des modifications introduites par cette édition, voir la contribution d'Ulla Kölving, à l'adresse http://c18.net/vo/vo_textes_siecle.php?div1 $=45$.

${ }^{48}$ Au sujet de ce changement, voir F. Brunot (1966, p. 961-964).
} 
Le second changement, relatif au doublement de la consonne «m », s'affirme de manière plus diffuse dans les écrits de Condorcet. Le «m simple» est omniprésent dans les manuscrits des années 1760 et du début des années 1770 ; il disparaît presque complètement à partir du début des années $1780^{49}$. Sachant par ailleurs que les manuscrits des années 1760 qui sont parvenus jusqu'à nous sont peu nombreux, on peut par exemple en déduire que toute pièce où le « $\mathrm{m}$ simple » domine très largement a été manifestement écrite au début des années 1770 ou, avec une probabilité plus faible, durant les années 1760 .

Grâce à la prise en compte de ces deux types de changement, la datation de plusieurs dizaines de manuscrits de Condorcet a pu être précisée, tandis que les informations chronologiques fournies par l'examen du contenu même de ces manuscrits étaient dans certains cas inexistantes $^{50}$. Ceci a parfois permis de mieux appréhender la genèse de la pensée de Condorcet, en attribuant des bornes chronologiques fiables à plusieurs de ses manuscrits ${ }^{51}$.

\section{Le support rédactionnel}

« Il [Condorcet] saisit tous les papiers qui se trouvent à portée de sa main, quelles que soient leur couleur, leurs dimensions, leur origine. Il écrit sur des billets d'invitation, sur des lettres, sur des factures, sur des feuillets qu'il a déjà couverts de chiffres : peu lui importe ». Cette affirmation de L. Cahen (1904, p. 781) repose sur l'hypothèse selon laquelle les types de papier de Condorcet qui sont parvenus jusqu'à nous, constituant environ 10000 feuillets, sont assez représentatifs de l'intégralité de ceux dont il a usé tout au long de sa vie ${ }^{52}$. Mais, en admettant même cette hypothèse, il demeure que le propos de L. Cahen doit être nuancé à plusieurs égards.

On remarquera d'abord qu'il ne saurait s'étendre à sa correspondance, si l'on excepte les brouillons de lettres. Comme ses contemporains, Condorcet utilise le plus souvent, pour

\footnotetext{
49 Parmi les manuscrits et sans prise en compte des passages raturés, la dernière occurrence de la graphie « home » que nous avons pour le moment localisée se trouve dans une lettre de Condorcet à Euler (14 fév. 1784, Archives de l'Académie des sciences de Saint-Pétersbourg, F. 1, op. 3, n 68, 1. 48). Si l'on tient compte des passages raturés, on trouve deux occurrences de la graphie « homes » (et aucune d' « home ») dans le Tableau historique de 1793-1794, contre un peu plus d'un millier d'occurrences de «homme(s) ». Voir le Prospectus (BI, Ms 885 (III), f. 386 v, 489 v (fin)) - ces deux passages n'ont pas été sélectionnés dans TH, p. 249, 421.

${ }^{50}$ La datation de la dizaine de Textes isolés d'un tableau historique des progrès de l'esprit humain (1770-1780) est un cas exemplaire. Jointe à une analyse du papier, cette datation a ainsi pu être établie par un examen des graphies, alors que le contenu principalement philosophique des textes en question ne livrait pas ou guère d'indices chronologiques. Sur ce point, voir N. Rieucau (2004 a).

${ }^{51}$ Nous pensons en premier lieu à ceux consacrés à l'arithmétique politique, au tournant des années 1760-1770, datés d'après l'examen de leurs graphies en « oi » ou en «ai ». Ces manuscrits ont été publiés par B. Bru \& P. Crépel (BC, chap. I et II).

${ }_{52}$ Pour une analyse générale de l'utilisation par Condorcet de son papier, voir C. Bustarret (2004), (2012, passim).
} 
ses lettres originales, du papier plié in $-4^{\circ}$, relativement fin, et souvent à la mode hollandaise ${ }^{53}$. Quant aux manuscrits, il convient de distinguer dans l'affirmation de L. Cahen deux catégories de supports d'écriture. La première est celle des papiers vergés ou vélins initialement vierges. Le propos de L. Cahen est, en ce cas, assez pertinent. En effet, Condorcet n'accorde visiblement aucune importance au type de papier sur lequel il écrit, à la différence de certains de ses proches, comme Sophie de Grouchy, ou de Rousseau. Il est ainsi courant de constater la présence, dans ses manuscrits, de plusieurs types distincts de papiers pour un même texte ${ }^{54}$. Un cas extrême est fourni par le brouillon autographe du Discours préliminaire sur l'histoire des sciences (1772) dont les neufs feuillets (BI, Ms 865, f. 220-228)ne comportent pas moins de six types différents, sous des pliages au demeurant non homogènes ${ }^{55}$. La seconde catégorie à laquelle L. Cahenfait allusion est celle des réemplois, c'est-à-dire l'utilisation de papiers ayant déjà servi comme support d'écriture ou d'impression. Le propos de L. Cahen pêche ici carrément par outrance, dans la mesure où les réemplois occupent certainement moins de $5 \%$ de l'ensemble des papiers utilisés par Condorcet. Ils ne semblent pas s'être multipliés pendant la période révolutionnaire, durant laquelle le prix du papier s'est pourtant considérablement accru du fait, principalement, de l'élévation de sa demande, elle-même due à l'essor de la presse périodique. Y compris pendant la dernière année de sa vie passée dans la clandestinité et alors même que ses biens et ses titres sont confisqués, Condorcet ne paraît pas avoir subi de réelles contraintes financières qui l'auraient obligé à multiplier les réemplois.

Qu'il s'agisse de réemplois ou de papiers initialement vierges, l'examen des supports utilisés par Condorcet se révèle en tout état de cause précieux en matière de datation. En ce qui concerne les réemplois, la présence d'une date sur ceux-ci - ce qui est fréquemment le cas quand Condorcet utilise comme support une lettre qu'il a reçue - fournit un terminus ante quem non à l'écriture de son manuscrit. Cet élément a permis de proposer ou de préciser une datation, voire de remettre en cause celle jusqu'alors admise, pour plusieurs textes de Condorcet relevant des mathématiques ${ }^{56}$ ou des sciences morales et politiques ${ }^{57}$.

\footnotetext{
${ }^{53}$ Sur ce point, voir C. Bustarret (2012, p. 61).

${ }^{54}$ Pour une illustration relative au Tableau historique des progrès de l'esprit humain, voir C. Bustarret (2004, p. 1214-1216).

${ }^{55}$ Voir ibid., p. 1214 ainsi que N. Rieucau (2004 b, p. 1144-1145).

${ }^{56}$ Voir par exemple B. Bru \& P. Crépel (1994, p. 575) au sujet de la Note sur la Préface de N. Bernoulli (17851786, BI, Ms 884, f. 1-2). On peut citer aussi le cas d'un Fragment sur le calcul intégral (fin 1792-1793, BI, Ms 876, f. 84 r) rédigé sur l'enveloppe d'une lettre à Condorcet, ce dernier étant désigné comme «Député à La Convention »: l'usage de ce réemploi prouve que les travaux de Condorcet sur le calcul intégral se sont poursuivis pendant la Révolution française, contrairement à ce qui a toujours été admis. Indiquons enfin que dans les papiers de Condorcet conservés à la Bibliothèque de l'Institut, on relève d'autres pièces datées sur lesquelles il a consigné des calculs de toutes sortes. Nous renvoyons par exemple, en nous en tenant à
} 
L'analyse du papier initialement vierge a quant à elle permis à ce jour de dégager deux éléments marquants en matière de datation. L'un est associé à l'utilisation par Condorcet de papier de fabrication picarde ; l'autre est la connaissance de la date de fabrication du papier.

Le premier de ces éléments dérive du fait que jusqu'à la fin de l'année 1775, et en particulier durant les périodes de vacances de l'Académie des sciences ${ }^{58}$, Condorcet se rendait fréquemment chez sa mère à Ribemont, dans l'Aisne ${ }^{59}$, avant que celle-ci ne le rejoigne à Paris, en compagnie de son oncle, Claude-Nicolas Gaudry. La maison familiale fut vendue en mars 1781 par ce dernier ${ }^{60}$ : il est possible que Condorcet s'y soit rendu, de temps à autre, jusqu'à cette date, même si nous n'avons pas trouvé d'indicesà ce sujet. Or le papier utilisé par les écrivains parisiens n'était pas, à cette époque, de fabrication picarde ; il provenait le plus souvent d'Auvergne, du sud-ouest de la France ou de Hollande ${ }^{61}$.On a donc tout lieu de penser que l'emploi par Condorcet de papier picard est associé à des séjours réguliers dans sa région natale jusqu'à la fin de l'année 1775 ou, moins probablement, lors de brefs passages dans cette même région jusqu'au début de l'année 1781. Dans les deux cas, on ne doit pas exclure qu'en dépit de sa médiocre qualité, Condorcet ait rapporté des feuillets de ce type de papier à Paris, où il les aurait utilisés. L'examen des types de papier relevés dans le corpus de ses manuscrits conforte ces deux hypothèses : la majeure partie des textes rédigés sur du

l'indication des cotes de ces pièces, aux Ms 855, f. 90 ; Ms 865, f. 250-251; Ms 867, f. 24 et 45-45 bis ; Ms 874, f. 89 ; Ms 876 , f. $79-80$.

${ }^{57}$ Voir N. Rieucau (2009, p. 47-49). Les textes concernés sont notamment la Vie de Voltaire - parfois datée de 1787 alors que l'un de ses feuillets manuscrits (BI, Ms 855, f. 523 v) date du 19 août 1788 - et deux manuscrits inédits, les Principes relatifs à la conversion d'un impôt indirect en un impôt direct et le Fragment sur les assignats, comportant deux feuillets où figurent respectivement les dates du 21 novembre 1784 (BI, Ms 2466, f. 88 v) et du 9 avril 1791 (BI, Ms 865, f. 152 v). Outre la Vie de Voltaire, d'autres textes fameux de Condorcet ont été rédigés sur des manuscrits comportant un ou plusieurs feuillets datés. Voir, par exemple, parmi les manuscrits de la Bibliothèque de l'Institut, l'Essai sur les Assemblées provinciales (dates des 5 et 27 août 1787, respectivement au Ms 856, f. 85 v et 86 v), le « Premier » des Mémoires sur l'instruction publique (date du 15 janv. 1791 au Ms 872, f. 418 v) et l'Esquisse (nommée «Prospectus ») d'un tableau historique des progrès de l'esprit humain (date du 4 mai 1793 au Ms 885-III, f. 434-437).

58 « Les vacances de l'Académie commenceront au huitième de septembre et finiront le onzième de novembre ; elle vaquera en outre pendant la quinzaine de Pâques, la semaine de Pentecôte, et depuis Noël jusqu'aux Rois », Règlement ordonné par le Roi pour l'Académie royale des sciences (26 janv. 1699, p. 410).

${ }^{59} \mathrm{La}$ dernière trace d'une présence de Condorcet à Ribemont date du 10 octobre 1775 , date à laquelle il écrit depuis son village natal à Turgot $(\mathrm{CH}$, p. 252-254). Le 17 du même mois, Condorcet ne semble toujours pas rentré à Paris, comme on le déduit à la lecture de la lettre que lui écrit ce jour-là Julie de Lespinasse (JNP, p. 124-125). On peut présumer que son séjour ne s'est pas prolongé jusqu'au 15 novembre. D'une part, en effet, par une lettre à Turgot du 15 octobre, Condorcet annonçait espérer voir son ami «à la Saint-Martin » (CH, p. 259), c'est-à-dire le 11 novembre. D'autre part, sa présence à Paris le 15 novembre est fort vraisemblable puisqu'il s'agit de la date de la séance de rentrée de l'Académie des sciences - mais nous n'avons pu vérifier cette présence d'après le Plumitif et le Procès-verbal relatifs à cette séance car la liste des académiciens y ayant participé n'y est pas relevée. On connaît, enfin, une lettre de Condorcet à Trudaine (AN, F 14/610) écrite depuis Paris le lendemain.

${ }^{60}$ Sur ce point, voir B. Vinot (1989 a, p. 417).

${ }^{61}$ Pour un aperçu général de la provenance des papiers consommés en France au XVIII ${ }^{\mathrm{e}}$ siècle, voir C. Bustarret (2012, p. 46-51). 
papier picard datent d'avant le milieu des années 1770 ; tandis que les dernières occurrences repérées datent de la seconde moitié de l'année 1778, voire du début de l'année $1779^{62}$. On aboutit par conséquent à la conclusion suivante : un manuscrit dont le support d'écriture est du papier picard (BIF 27, 47, 59, 94) doit être daté, avec une grande vraisemblance, d'une époque antérieure à la fin de l'année 1775 et, avec une probabilité plus faible, de l'intervalle 1776-1781. Dans les deux cas, ce manuscrit a pu être rédigé durant les périodes de vacances de l'Académie des sciences.
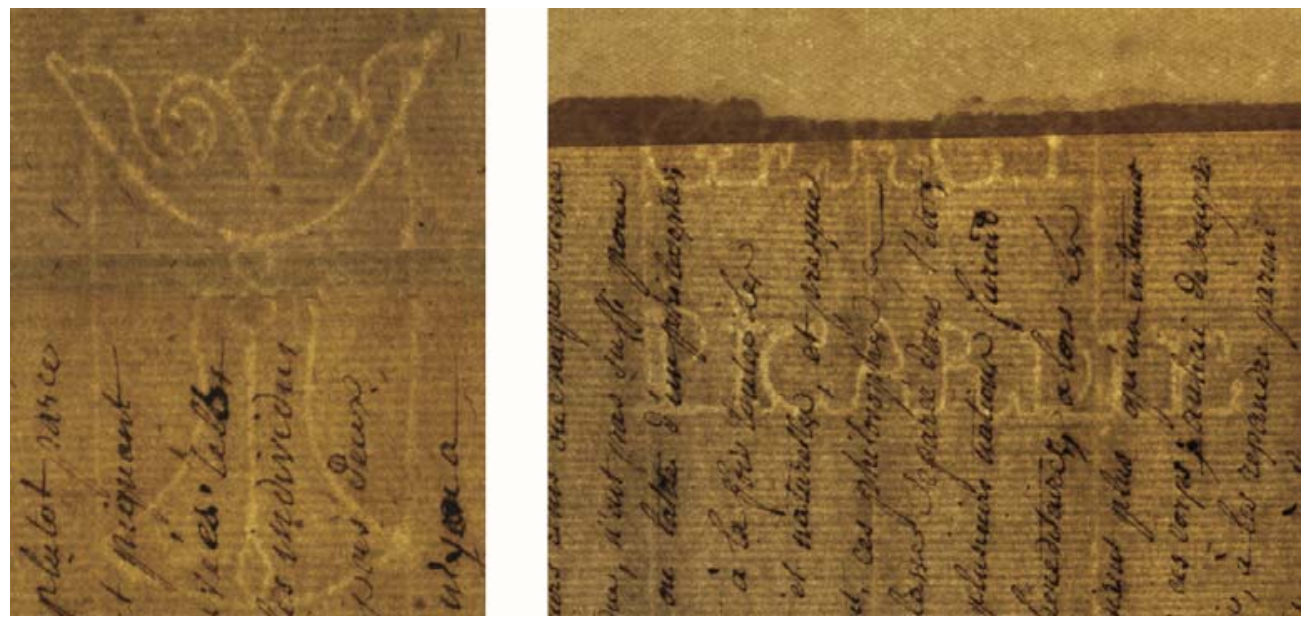

Document 3 : Filigrane de type BIF 27. Marque : Cloche couronnée ; contremarque (ici tronquée) : « J [cœur] L [cœur] LOUBRY / GERCY / PICARDIE $»^{63}$.

S'agissant du deuxième élément de datation provenant de l'analyse du papier initialement vierge, la présence de filigranes millésimés a été relevée dans les papiers de Condorcet pour les années suivantes : 1778 (BIF 86), 1779 (BIF 95), 1780 (BNF 365), 1783 (BIF 61) et 1789 (BIF 9) ${ }^{64}$. En outre, on sait qu'un autre type de papier a été fabriqué sur la période 1786-1789 (BIF 15). Or, la durée maximale pendant laquelle Condorcet use de son papier après l'avoir acquis est, le plus souvent, de cinq années environ. Si un manuscrit comporte un papier millésimé, on en déduit donc que sa rédaction ne peut être à l'évidence

\footnotetext{
${ }^{62}$ On les trouve sur le manuscrit autographe de l'Éloge de Linné (BI, Ms 855, f. 65-89; Ms 873, f. 339-353). Carl von Linné est mort le 10 janvier 1778 mais l'éloge de Condorcet ne fut prononcé à l'Académie des sciences que le 14 avril 1779. Par ailleurs, une lettre de Condorcet à Linné fils, datée du 15 août 1778 (Londres, Linnean Society, Linnean Correspondence, vol. 3, f. 89-90), confirme qu'il n'avait alors pas achevé, ni peut-être même commencé, la rédaction de son éloge.

${ }^{63}$ La marque et la contremarque proviennent de deux feuillets de la copie du Discours préliminaire sur l'histoire des sciences (1772; BI, Ms 865, f. 234 v-235 r, 231 v). Nous avons réalisé ces clichés (ainsi que ceux du Document 4, infra, p. 16), avec C. Bustarret et G. Coutagne, à l'aide d'une feuille souple à éclairage par fibres optiques faisant apparaitre la trame et le filigrane du papier.

${ }^{64}$ On doit exclure le faux millésime « $1742 »$ dont on trouve de nombreuses occurrences dans les papiers de Condorcet (par exemple BIF 5, 20, 22, 24). Cette mention est liée à un arrêt imposant aux fabricants de papiers, en septembre 1741, d'indiquer son année de fabrication dans le filigrane à partir du $1^{\text {er }}$ janv. 1742. Certains papetiers continuèrent toutefois, pendant plusieurs dizaines d'années, de reproduire le millésime « 1742 ». Sur ce point, voir R. Gaudriault (1995, p. 27).
} 
antérieure à l'année indiquée par le filigrane, qu'elle coïncide avec cette année ou lui est, au maximum, fort vraisemblablement postérieure de cinq années environ.
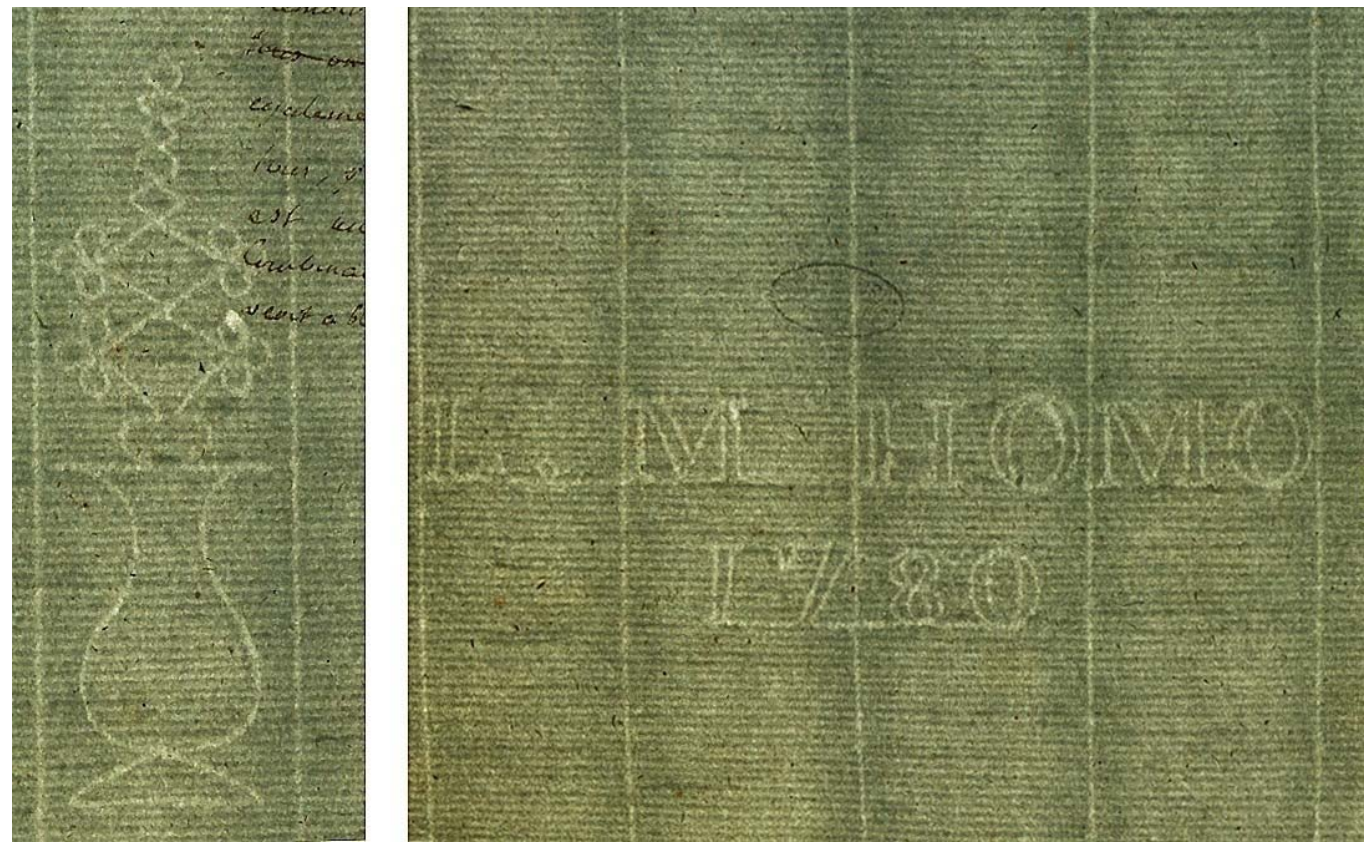

Document 4 : Filigrane de type «BNF $365 »$. Marque : Pot surmonté d'uncroisillon fleuronné ; contremarque : «L. M. HOMO / $1780 »^{65}$.

Le repérage de papier picard ou de papier dont on connaît la date de fabrication a permis d'établir, d'améliorer ou de corriger la datation de nombreux manuscrits de Condorcet, publiés ou inédits ${ }^{66}$.

Mais il convient de garder à l'esprit tout l'intérêt que représente par ailleurs la datation par recoupement, consistant à déceler une coïncidence entre le type de papier d'une pièce datée ou datable et celui d'une pièce non datée. Cette méthode a été en particulier utile lors de deux analyses génétiques. Elle a permis d'une part d'associer entre eux divers manuscrits duTableau historique des progrès de l'esprit humain, au rang desquels figuraient un Plan en neuf époques et le brouillon - intitulé «Prospectus»-de l'Esquisse ${ }^{67}$. D'autre part, cette méthode a étayé le fait que le Tableau général de la science avait été rédigé dans l'urgence par Condorcet en juin 1793, sans qu'il puisse en corriger les épreuves, étant sur le point

\footnotetext{
${ }^{65}$ La marque et la contremarque sont issues de deux feuillets d'un manuscrit intitulé Génie (début des années 1780 ; BI, Ms 865, f. 398 r et 399 v).

${ }^{66}$ Certains résultats ont été publiés dans C. Bustarret (2004, p. 1216-1219), N. Rieucau (2004 a, p. 179-183), (2009, p. 46-49). Nous n'en n'évoquerons qu'un, peut-être le plus éloquent, associé aux Fragments sur la liberté de la presse. Ce texte est daté de 1776 dans les Euvres de Condorcet (OC, t. XI, p. 253-314), date qui a été retenue par les commentateurs. Seulement, le filigrane de plusieurs de ses feuillets manuscrits (BI, Ms 857, f. 2037) comportent le millésime « $1779 »$. Cet indice, enrichi par l'examen des graphies et une reconsidération du contenu du texte, a permis d'établir une date de rédaction située aux alentours de 1781.

${ }^{67}$ À ce sujet voir C. Bustarret (2004, p. 1216-1217) ainsi que P. Crépel, Y. Garlan \& N. Rieucau (2004, p. 1053).
} 
d'entrer dans la clandestinité ${ }^{68}$. Il en a résulté une version imprimée multipliant les erreurs typographiques et les imprécisions, ce qui a grandement contribué à la mauvaise réception du fameux projet de Mathématique sociale présenté par Condorcet dans ce texte, daté de façon fantaisiste par la plupart de ses éditeurs et commentateurs. Dans les deux cas, la datation par recoupement, établie à partir d'indices matériels, a ainsi permis de reconsidérer l'analyse qui été faite du contenu même de l'œuvre de Condorcet.

\section{Les cachets de cire}

Un peu plus d'une centaine de cachets dont le motif est identifiable ont été relevés sur les presque 600 lettres originales de Condorcet que nous connaissons pour le moment ${ }^{69}$. Dans deux cas sur trois environ, le motif repéré correspond aux armes de la famille paternelle de Condorcet, les Caritat, soit d'azur, au dragon ailé d'or armé et lampassé de sable ${ }^{70}$. L'utilisation de ce cachet est attestée dans une grande partie de la correspondance de Condorcet, de ses premières lettres des années 1760 jusqu'à la Révolution. L'ultime trace connue figure dans une lettre à Brissot du 31 décembre $1789^{71}$.

Il s'agit là, fort vraisemblablement, d'une des dernières utilisations par l'académicien de ce sceau. En effet, l'abolition de la noblesse héréditaire, des titres, ordres militaires, armoiries et autres livrées par la Constituante, le 19 juin 1790, l'a sans doute contraint à n'en plus faire usage, quoiqu'il critiquât cette abolition parce que, selon lui, «l'assemblée nationale aurait du non defendre de porter des armoiries [...] mais declarer que les armoiries [...] cesseraient d'être des attributs distinctifs appartenans à une famille particuliere $»^{72}$. D'ailleurs, tout en s'opposant pour la même raison à l'interdiction d'user de titres ajoutés aux noms de famille ${ }^{73}$, Condorcet - sans aller jusqu'à se donner le nom de Caritat tout court cessa systématiquement de s'attribuer la dignité de marquis ${ }^{74}$, dans sa correspondance, dès août $1789^{75}$, ce qui peut au demeurant constituer un indice de datation de celle-ci ${ }^{76}$.

\footnotetext{
${ }^{68}$ Voir P. Crépel \& N. Rieucau (2005, p. 245-249).

${ }^{69}$ Nombreux sont les cachets aujourd'hui absents ou bien impossibles à décrire car ils sont trop écrasés, à moins qu'il n'en subsiste que quelques fragments voire de simples traces.

${ }^{70}$ Une reproduction de ce motif se trouve dans les Armoiries de quelques familles de Ribemont (s. d., AD Aisne, Fonds Amédée Piette, 42 fi 68) et dans C.-R. d'Hozier (1697-1709, Fr. 32238 : vol. XI, p. 140). Voir aussi A.-M. Chouillet \& P. Crépel (dir.) (1997, p. 19).

${ }^{71} \mathrm{AN}, 446 \mathrm{AP} / 7$, pièce 78.

${ }^{72}$ Sur le décret du 19 juin 1790, BI, Ms 860, f. 271 r.

${ }^{73}$ Outre le manuscrit précédemment cité, voir Condorcet à La Métherie (juin 1790, OC, t. I, p. 327).

${ }^{74}$ Soulignons que ce titre, souvent considéré comme acquis par les commentateurs, ne repose en réalité sur aucun document connu. Il en va de même de la dignité de «baron» dont le jeune Condorcet s'affubla au tout début des années 1760. Voir Plumitif des séances de l'Académie des sciences (23 déc. 1761) et Interscendentes (2 juil. 1762, Londres, Wellcome Library, Ms 8954/4, f. 2 r).

${ }^{75}$ Voir l'édition originale de sa lettre ostensible, du 30 août 1789, au comte Mathieu de Montmorency.
} 
L'hypothèse d'après laquelle Condorcet aurait abandonné en 1790 l'usage de la matrice représentant les armes de sa famille paternelle est renforcée par le fait suivant : si, sur la période 1790-1794, un seul motif de cachet de cire a, en l'état actuel de nos recherches, été identifié, il ne correspond précisément pas à la matrice précédemment décrite. Cet apax, relevé sur une lettre de Condorcet adressée à Brissotvraisemblablement en octobre $1791^{77}$, est constitué par la mention «La Vérité », en référence à leur appartenance commune, à cette époque, à la Société des amis de la vérité.

Outre la matrice de sceau représentant les armes de sa famille paternelle, on doit signaler, au deuxième rang de fréquence des matrices utilisées par Condorcet, celle composée de deux écus ovales (Document 5), à dextre aux armes des Caritat et à senestre aux armes de sa famille maternelle, les Gaudry, soit des armes d'azur à une tour d'argent maçonnée de sable, accompagnée d'un croissant d'or en $\operatorname{chef}^{78}$ : la plupart des emplois de cette matrice interviennent jusqu'au milieu des années 1770, lorsque Condorcet séjourne chez sa mère, à Ribemont, ayant alors visiblement omis de prendre par devers lui sa matrice de sceau habituelle.

\footnotetext{
${ }^{76}$ On notera toutefois que dans deux Certifications de conformité, respectivement du 2 septembre 1789 et du 29 janvier 1790 (AAS, Pochettes de séance du 29 août 1789 et du 27 janv. 1790), Condorcet se donne encore le titre de marquis. Pour autant que nous le sachions, la seconde de ces pièces est la dernière où il signe de la sorte. Il continue néanmoins de maintenir la particule « de » dans ses manuscrits, qu'il s'agisse de lettres personnelles ou de documents académiques. La première occurrence de «Condorcet» sans la particule que nous connaissions se trouve dans une Certification de conformité du 21 août 1790 (AAS, Pochette de séance du 4 août 1790), mais on note encore, au 7 septembre de la même année, la signature « de Condorcet» dans un Rapport sur un mémoire de Trouville (AAS, Pochette de séance du 7 sept. 1790). Cette signature, sauf erreur de notre part, ne se retrouvera plus par la suite. Le relevé de sa présence ou de son absence peut donc aussi constituer un élément de datation.

${ }^{77}$ Paris, Musée des Lettres et Manuscrits, pièce n ${ }^{\circ} 43826$.

${ }^{78}$ On trouve une reproduction de ce motif dans les Armoiries de quelques familles de Ribemont (s. d., AD Aisne, 42 fi 68). Celle figurant dans C.-R. d'Hozier (1697-1709, Fr. 32253 : vol. XXXII, p. 32) est en revanche légèrement fautive.
} 


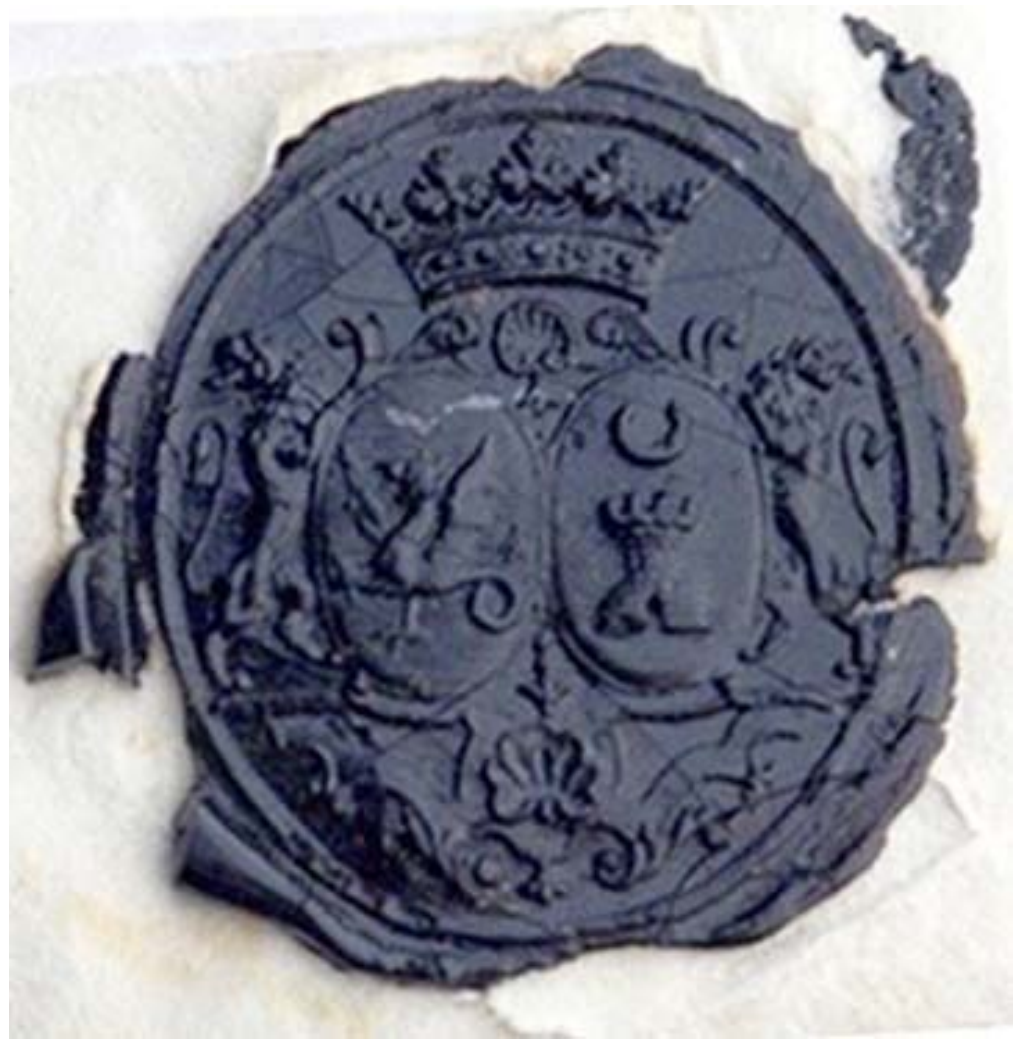

Document 5 : Cachet de cire noir composé de deux écus ovales, dans Condorcet à Turgot (fin mai 1774), BI, Ms 7442, f. 45 v.

C'est également depuis son village natal que Condorcet a adressé une lettre à Turgot, le 10 mai 1774, où est apposé le cachet d'une matrice représentant un personnage ailé tenant une coupe ${ }^{79}$. Il s'agit de la seule occurrence de ce motif de cachet que nous connaissions. Nous ignorons sa signification exacte mais sa présence s'explique, peut-être, par le fait que Condorcet ne dispose alors d'aucune matrice de sceau, parmi celles précédemment évoquées, liées à son ascendance familiale. La même raison nous paraît avoir motivé l'utilisation d'une autre matrice, repérée par un cachet constitué de diverses boucles entrelacées, motif dont nous ne connaissons pas non plus le sens précis. Ce cachet se trouve sur une lettre de Condorcet à de Lisy de $1787^{80}$, tandis qu'il séjourne près de Meulan, au Château de Villette, propriété de la famille de Grouchy.

On doit aussi citer l'emploi par Condorcet, du temps de la période active (1776-1791) de son secrétariat à l'Académie royale des sciences, de la matrice représentant les armes de ladite, soit un sceau d'azur à un soleil d'or accompagné de trois fleurs de lis du même, deux en chef et une en pointe ${ }^{81}$. Le cachet de cette matrice apparait sur les lettres que Condorcet

\footnotetext{
${ }^{79}$ Localisation inconnue-vente de Proyart, 60/35, coll. privée.

${ }^{80}$ BI, Ms 865, f. 457 r-v.

${ }^{81}$ Différentes versions de ce motif se trouvent dans J. Tremblot (1950).
} 
expédie au titre de cette institution - par exemple les notifications officielles rédigées sur du papier pré-imprimé pour informer un savant étranger de sa nomination comme correspondant $^{82}$ - mais pas de façon systématique. D'une part, en effet, Condorcet use parfois de cette matrice pour des courriers personnels, ce qui est attesté par deux lettres adressées aux époux Suard ${ }^{83}$. La datation peut alors être étayée par le recours au calendrier deses présences à $1^{\prime}$ Académie des sciences $^{84}$, puisqu'il est raisonnable de penser, du moins en première hypothèse, que Condorcet ne s'est servi de la matrice en question que dans les bâtiments de cette institution. D'autre part, Condorcet peut aussi écrire en tant que Secrétaire perpétuel mais ne pas utiliser la matrice de l'Académie, manifestement parce qu'il ne s'y trouve pas : on citera par exemple une lettre du 20 novembre 1776, où il déclare à Anisson-Duperron être «chargé par l'académie » de lui écrire, mais où il utilise une matrice représentant un pot fleuri ${ }^{85}$. Cette même matrice, au motif manifestement banal, a été repérée par trois autres cachets sur des lettres également rédigées en 1776: l'une adressée à Stanhope le 19 novembre ${ }^{86}$ - soit la veille de la rédaction de sa lettre à Anisson-Duperron ${ }^{87}$ - sans que 1'on sache où Condorcet se trouve ; une autre envoyée à Turgot au début de l'été, où il indique résider dans sa maison de Nogent ${ }^{88}$; une dernière à l'attention de Fabroni, le 14 février, où il mentionne seulement être à Paris ${ }^{89}$.

L'emploi d'une dernière matrice, s'il ne doit pas non plus être associé à la présence de Condorcet en un lieu précis, paraît cependant avoir aussi été relativement circonscrit dans le temps, ce qui peut donc là encore se révéler utile en matière de datation. Cette matrice représente une effigie, visiblement d'un personnage de l'Antiquité ; son cachet se trouve sur une lettre à Turgot du 4 décembre $1773^{90}$.

Concernant les couleurs des cachets ou des traces de cachets pour le moment répertoriées, on constate qu'une vingtaine sont noires, tandis qu'un peu plus de 200 sont rouges. Condorcet use notamment de cire noire lors d'une période de deuil, comme d'autres

\footnotetext{
${ }^{82}$ Voir Condorcet à de Sivry (21 août 1783, Archives de l'Institut d'histoire de Saint-Pétersbourg, K 11, pièce 49) ou encore Condorcet à Thunberg (5 sept. 1787, Bibliothèque nationale de Russie, F. 993 - op. 2, carton 36, $\mathrm{n}^{\circ}$ 1374). Le texte imprimé s'achève au demeurant comme suit: «En foy de quoy j'ai signé les présentes, auxquelles j'ai apposé le sceau de l'Académie. »

${ }^{83} \mathrm{BnF}$, Condorcet à A. Suard (juin-juil. 1780, NAF 23639, f. 25 v) ; Condorcet à J.-B. Suard (fin mai 1782, ibid., f. $36 \mathrm{v}$ ).

${ }^{84} \mathrm{Ce}$ calendrier est disponible à l'adresse http://inventaire-condorcet.com/fra/page/11.

${ }^{85}$ Condorcet à Anisson-Duperron (20 nov. 1776, Saint-Pétersbourg, Archives de l'Institut d'histoire, K 11, pièce $52)$.

${ }^{86}$ Condorcet à Stanhope (19 nov. 1776), Maidstone, Center for Kentish Studies, U1590, C 13/13.

${ }^{87}$ Ces deux lettres sont d'ailleurs rédigées sur le même type de papier (BIF 40).

${ }^{88}$ Condorcet à Turgot (début de l'été 1776), localisation inconnue-vente de Proyart, 58/35, coll. privée.

${ }^{89}$ Condorcet à Fabroni (14 fév. 1776), Pise, Biblioteca Universitaria, Ms 422.26/45.

${ }^{90} \mathrm{BI}$, Ms 7442, f. 35 v.
} 
épistoliers de son temps ${ }^{91}$. Ainsi, le cachetage noir de sa lettre à d'Alembert ${ }^{92}$, rédigée vers septembre 1768, marque le deuil de la reine Marie Leszczynska, survenue le 24 juin de la même année. Condorcet utilise pareillement de la cire noire pour cacheter son courrier pendant les 6 mois qui suivent la mort de Louis XV, le 10 mai 1774 - indice utile pour faciliter en particulier la datation de ses lettres à Turgot, du temps du ministère (août 1774mai 1776) de ce dernier ${ }^{93}$. Condorcet a également recours à de la cire noire pour marquer le deuil d'un membre de sa famille. Il en va ainsi dans les semaines qui suivent la disparition de son oncle et parrain, Claude-Nicolas Gaudry, le 19 juillet 1784.

Si la prise en compte de la couleur de la cire peut donc être utile lorsqu'on est confronté à des lettres non datées, il convient néanmoins de manier cet indice avec prudence. À l'instar de l'usage d'un type de papier, celui d'une cire de telle ou telle couleur peut être dû à une raison purement pragmatique, comme un simple hasard dans le stockage ou à un défaut dans l'approvisionnement. Il faut aussi considérer que cette observation du deuil par Condorcet peut être suivie avec plus ou moins de rigueur selon la nature de sa correspondance, c'est-à-dire le type de lettre (un billet remis en mains propres, une lettre officielle rédigée en tant que Secrétaire de l'Académie, une lettre personnelle...) ou l'identité du destinataire (un proche, une sommité, un étranger...). Ces divers éléments peuvent ainsi expliquer certaines occurrences de couleur de cire isolées, comme le noir dans deux lettres du printemps $1775^{94}$ ou dans une lettre de février $1791^{95}$.

\section{CONCLUSION}

La perspective temporelle dans laquelle s'inscrit l'œuvre de Condorcet détient un statut ambivalent. Souvent présenté comme un auteur situant son propos dans le long terme, ce que la fameuse « Dixième époque » de son Tableau historique illustre on ne peut mieux, ses écrits sont simultanément, si l'on excepte ceux de mathématiques pures, en prise quasi constante

\footnotetext{
91 En la matière, une étude spécifiquement consacrée à d'Alembert sera disponible dans la synthèse pour l'Habilitation à diriger des recherches d'I. Passeron (CNRS-Observatoire de Paris).

92 Ms 876, f. 2-3.

${ }^{93} \mathrm{La}$ datation de l'intégralité de la correspondance Condorcet-Turgot, corrigeant celle de l'édition de Charles Henry (1883), figurera bientôt sur le site de l'Inventaire Condorcet.

${ }^{94}$ Condorcet à Turgot (début mars 1775, localisation inconnue-vente de Proyart, 68/35, coll. Privée), Condorcet à Trudaine (mars 1775, AN, F 14/610). Dans le cas présent, et même si des lettres de la même époque sont cachetées à la cire rouge, l'usage de cire noire s'expliquerait par le fait que Condorcet en a encore à sa disposition, après avoir observé l'année précédente le deuil de Louis XV.

${ }^{95}$ Condorcet à Brissot (26 fév. 1791, AN, $446 \mathrm{AP} / 7$, pièce 74).
} 
avec l'actualité de son temps: l'affirmation de la "Dixième époque », en réponse à la dictature montagnarde, en est elle-même une manifestation majeure ${ }^{96}$. La datation la plus précise possible de ces textes, à commencer par ceux qui ont été rédigés de la veille de la Révolution jusqu'à la proscription de Condorcet, se révèle donc très fréquemment d'une importance fondamentale. Tenter d'attribuer une date de rédaction à la plupart des lettres de Condorcet constitue, en outre, une entreprise dont il n'est pas la peine de justifier l'intérêt. Face à de telles tâches, l'analyse matérielle est d'un grand secours.

L'intérêt des données codicologiques pour inscrire l'œuvre de Condorcet dans le temps est, aussi, un plaidoyer pour la publication la plus fidèle possible à son état manuscrit. Le respect des graphies, la présence d'un descriptif détaillant notamment la nature du papier, l'espace graphique et les écritures sont, à ce titre, autant d'éléments qui, selon nous, mériteraient de figurer dans toute édition scientifique des écrits de l'académicien ${ }^{97}$. Cet impératif doit s'étendre aux manuscrits dont on connaît la date de rédaction. Leurs caractéristiques matérielles constituent en effet un lot d'indices qui, par recoupement, peuvent servir ultérieurement à l'établissement d'une période de rédaction à des manuscrits non datés, qu'il s'agisse de lettres, d'avant-textes de toutes sortes ou de documents réellement inédits.

En ce sens, gageons que plus le relevé des caractéristiques matérielles de pièces datées ou non s'enrichira - par un inventaire croissant des filigranes, un travail plus détaillé sur l'évolution de l'écriture et des graphies de Condorcet, sur la nature des encres, l'espace graphique de ses manuscrits ou encore l'établissement d'une isographie exhaustive de ses copistes - plus il permettra d'aboutir à des résultats nombreux et précis, remettant parfois en cause, nous n'en n'avons pas crainte, certains de ceux esquissés ici, et permettant d'appréhender encore mieux l'œuvre et la vie de l'académicien.

\footnotetext{
${ }^{96}$ Sur ce point, voir N. Rieucau (2003, p. 32).

${ }^{97}$ L'idéal étant évidemment de les publier sous forme électronique, non seulement pour des raisons de souplesse et de lisibilité, mais également pour fournir le texte y compris dans une version modernisée, cela afin de faciliter la recherche de mots et l'analyse lexicométrique.
} 


\section{RÉFÉRENCES}

\section{ABRÉVIATIONS ET SIGLES UTILISÉS \\ DANS LE COURS DU TEXTE OU DE LA LISTE DE RÉFÉRENCES}

- AAS : Archives de l'Académie des sciences (Paris).

- AD : Archives départementales.

- AN : Archives nationales de France (Paris).

- $\quad$ BC : Condorcet - Arithmétique politique - textes rares ou inédits (1767-1789), B. Bru et P. Crépel (éd.), Paris :Ined, $1994^{98}$.

- BHVP : Bibliothèque historique de la ville de Paris.

- BI : Bibliothèque de l'Institut de France (Paris).

- BIF [+ nombre] et BNF [+ nombre] : codes d'identification des types de papiers dont la première occurrence a été trouvée, par C. Bustarret, à la Bibliothèque de l'Institut de France et à la Bibliothèque nationale de France.

- BnF : Bibliothèque nationale de France (Paris).

- BOP : Bibliothèque de l'Observatoire de Paris.

- CGM : Catalogue général des manuscrits des bibliothèquespubliques de France, Paris : Bibliothèque nationale : puis Éditions du CNRS, 1886-

- CH : Correspondance inédite de Condorcet et de Turgot 1770-1779, Ch. Henry (éd.), Paris : Charavay Frères, 1883.

- EB : Correspondanceinédite de Condorcet et Madame Suard (1771-1791), É. Badinter(éd.), Fayard, 1988.

- JNP : Julie de Lespinasse - Lettres à Condorcet, J. N. Pascal (éd.), Paris : Desjonquères, 1990.

- OC: Euvres de Condorcet, [Éliza O’Connor], FrançoisArago, Arthur O’Connor (éd.), Paris :Firmin-Didot, 1847-1849, 12 t.

- TH : Tableau historique des progrès de l'esprit humain 1772-1794, JeanPierreSchandeler\& Pierre Crépel(dir.), Paris :Ined, 2004.

\section{CONDORCET}

\section{Manuscrits}

\section{Types de papier}

NB : Ces types de papiers sont répertoriés dans la base de données « MUSE » conçue par C. Bustarret et S. Linkès. Les dimensions correspondent au format in-plano du papier.

- BIF 5 : vergé, azuré, assez fin $(107 \mu)$ et lisse. Hauteur x largeur (rogné) : 388x 534 mm. Marque : Oiseau dans un cercle. Contremarque: « $\mathrm{C}$ [cœur] PIGNION FIN / AUVERGNE 1742 ». Écart des lignes de chaînette : 27-28 mm.

- BIF 9 : vergé, légèrement azuré, assez épais (146 $\mu)$, assez lisse et de qualité supérieure. Hauteur x largeur : 352 x $576 \mathrm{~mm}$. Marque : initiales « G D »entourées de lauriers dans un

\footnotetext{
${ }^{98}$ Seules les références aux écrits de Condorcet (et non aux commentaires de B. Bru et P. Crépel) sont indiquées par cette abréviation.
} 
cercle / «F \&. N \&.». Contremarque : initiales « $\mathrm{G} D$ »entourées de lauriers dans un cercle/ «F\&.N\&. » / « 1789 ». Écart des lignes de chaînette : 28-30 mm.

- BIF 15 : vergé, écru, assez épais $(145 \mu)$ et légèrement rugueux. Hauteur x largeur : 336x $432 \mathrm{~mm}$. Marque : Couronne royale. Contremarque : «DLG / A / COURTALIN ». Écart des lignes de chaînette : 26-27 mm.

- BIF 20 : vergé, azuré, épais $(165 \mu)$ et assez lisse. Hauteur x largeur : 350 x 408 mm. Marque ?Contremarque : « C [cœur] PIGNION FIN / AUVERGNE 1742 ».Écart des lignes de chaînette : 27-28 mm.

- BIF 22 : vergé, écru, assez épais $(143 \mu)$ et assez lisse. Hauteur x largeur : 318 x $420 \mathrm{~mm}$. Marque :armes de Le Tellier simplifiées. Contremarque : monogramme de Le Tellier / «V [fleur de lis] BEAL FIN / AUVERGNE 1742 ». Écart des lignes de chaînette : 25,5-27 $\mathrm{mm}$.

- BIF 24 : vergé, écru, assez fin $(116 \mu)$ et lisse. Hauteur x largeur : 350 x $450 \mathrm{~mm}$. Marque : Griffon. Contremarque : «I [cœur] BOUCHET MOYEN / AUVERGNE 1742 ».Écart des lignes de chaînette : 26-27 mm.

- BIF 27 : vergé, écru grisé, moyennement épais $(124 \mu)$ et lisse. Hauteur x largeur : 394 x $572 \mathrm{~mm}$. Marque : Cloche couronnée. Contremarque : «J [cœur] L [cœur] LOUBRY / GERCY / PICARDIE ». Écart des lignes de chaînette : 26 -30 mm.

- BIF 40 : vergé, écru, moyennement épais $(129 \mu)$ et lisse. Hauteur x largeur : 310 x 394 mm. Marque : Cornet sur écu fleurdelisé / «D \& C BLAUW ». Contremarque « D \& C BLAUW ». Écart des lignes de chaînette : 24 -26 mm

- BIF 47 : vergé, écru, foncé en transparence, assez épais $(142 \mu)$ et légèrement rugueux. Hauteur x largeur : 400 x $572 \mathrm{~mm}$. Marque : Cloche ? Contremarque : « J [cœur] L [cœur] LOUBRY / GERCY / PICARDIE ». Écart des lignes de chaînette : 27-29 mm.

- BIF 59 : vergé, écru, assez épais $(143 \mu)$ et assez lisse/légèrement rugueux. Hauteur x largeur: 400 x $580 \mathrm{~mm}$. NB : forme «double» soit: Marque : Cloche couronnée. Contremarque : «I [point] L [point] LOUBRY / PICARDIE»+ Marque : Cloche couronnée. Contremarque : «I [point] L [point] LOUBRY : GERCY ». Écart des lignes de chaînette : 25-28 mm.

- BIF 61 : vergé, écru, épais $(164 \mu)$ et assez lisse. Hauteur x largeur : 324 x $420 \mathrm{~mm}$. Marque : Raisin / « $1783 »$. Contremarque : « M [point] LAPIERE / POITOU ». Écart des lignes de chaînette : $26-28 \mathrm{~mm}$.

- BIF 86 : vergé, écru, assez épais $(144 \mu)$ et rugueux. Hauteur x largeur : 314 x 390 mm. Marque : Pot surmonté de motif floral avec « $1778 »$ à l'intérieur du pot. Contremarque : «G. DE ROUEN»+ « N. » [ou « M. »] HERMIER [?]/ FIN ».Écart des lignes de chaînette : 23-26 mm.

- BIF 94 : vergé, écru, assez épais $(154 \mu)$ et assez lisse. Hauteur x largeur : 386 x 564 mm. NB : on distingue les empreintes de deux formes jumelles : dans l'une d'elles le deuxième cœur de la contremarque manque soit : Marque ?. Contremarque : « J [cœur] L LOUBRY / GERCY / PICARDIE » + Marque : ?. Contremarque : « J [cœur] L [cœur] LOUBRY / GERCY / PICARDIE ».Écart des lignes de chaînette : 24-28 mm.

- BIF 95 : vergé, légèrement azuré, assez épais $(141 \mu)$ et lisse. Hauteur x largeur : 315 x 396 mm. Marque : Pot surmonté d'un croisillon fleuronné. Contremarque : « C. L. S. / 1779 ». Écart des lignes de chaînette : 26-28 mm.

- BNF 365 : vergé, azuré, assez épais $(131 \mu)$ et légèrement rugueux. Hauteur x largeur : 310 x 490 mm. Marque : Pot surmonté d'un croisillon fleuronné.Contremarque : « L. M. HOMO / 1780 ». Écart des lignes de chaînette : 26-30 mm. 


\section{Textes}

- De l'influence de la Révolution d'Amérique sur l'Europe, 1786, BI, Ms 857, f. 530-561.

- [Dernière réflexion sur la procédure écrite ou parlée], 1791, BI, Ms 857, f. 13 ; dans G. Tarello (1975, p. 406-407).

- [Discours préliminaire sur l'histoire des sciences], [1772], BI, Ms 865 f. 220 r-228 v (autographe), Ms 865, f. 219 r-v, 229 r-235 r (copie); TH, p. 134-144.

- Élémens du calcul des probabilités et son application aux jeux de hasard, à la loterie et aux jugemens des hommes, [1786-1787], BI, Ms 865 f. 79-80, 82, 365-367 ; Ms $873 \mathrm{f}$. 300-304 ; Ms 875 f. 193-194 ; Ms 882 f. 154-163 = f. 14-23 ; Ms 883, f. 184, 189 bis, 191-204; BOP, Z 39 f. 1-6.

- Éloge d'Euler, 1785, BnF, NAF 27261, f. 1-33.

- Éloge de Linné, 1779, BI, Ms 855, f. 65-89 ; Ms 873, f. 339-353.

- Essai d'une méthode generale pour integrer les equationsdifferentielles a deux variables, AAS, Pochette de séance du 18 nov. 1761

- Essai sur la constitution et les fonctions des assemblées provinciales, 1788, BI, Ms 856.

- Essai sur l'application de l'analyse à la probabilité des décisions rendues à la pluralité des voix, 1785, BI, Ms 857, 865, 873-875 ; BOP, Z 29, passim.

- Exemple des méthodes tecniques, [1793-1794], BI, Ms 885 (I), f.120² r-129 v, TH, p. 1033-1048.

- $\quad$ [Fragments sur la liberté de la presse], [vers 1781], BI, Ms 857, f. 20-37 ; OC, t. XI, p. 253-314.

- $\quad$ [Fragment sur le calcul intégral], [fin 1792-1793], BI, Ms 876, f. 84.

- [Fragment sur les assignats], [9-26 avr. 1791],BI, Ms 865 f. 152-161 (autographe), f. 5258 (copie).

- Génie, [début des années 1780], BI, Ms 865, f. 398-399; TH, p. 727.

- Interscendentes, 2 juil. 1762, Londres, Wellcome Library, Ms 8594/4.

- Mémoire sur le calcul des probabilités, 1784-1787, BI, Ms 854, 865, 873, 875, passim ; BOP, Z 29, f. 2-6, 14-19 ; Bloomsbury Book Auctions, 28 nov. 1985, Sale 47, lot $\mathrm{n}^{\circ} 71$; BC (publ. partielle), p. 451-464.

- Mémoires sur l’instruction publique, 1791, BI, Ms 865, f. 407 ; Ms 872, passim ; Ms 884, 300-302, 304.

- $\quad$ [Note sur la préface de N. Bernoulli], [1785-1786], BI, Ms 884, f. 1-2 ; BC, p. 575-576.

- [Petit ouvrage "plus métaphysique que mathématique » sur le calcul des probabilités], 1773-1774, BI, Ms 875, f. 84-109 ; BC, p. 283-294.

- $\quad$ [Plan de 1793], BnF, NAF 4586, f. $1 \mathrm{r}$; TH, p. 1054.

- $\quad$ Principes relatifs à la conversion d'un impôt indirect en un impôt direct], [après le 21 nov. 1784], BI, Ms 2466, f. 88-89.

- Prospectus d'un tableau historique des progrès de l'esprit humain, 1793, BI, Ms 885 (III), f. 377 r-518 r ; TH, p. 233-459.

- [Rapport sur le choix d'une unité de mesure], 1791, BI, Ms 872, f. 601-602 (1 ${ }^{\text {ère }}$ version), 591-593 ( $2^{\mathrm{e}}$ version).

- [Rapport un mémoire de Trouville] (avec Meusnier, Monge et Vandermonde), 7 sept. 1790, AAS, Pochette de séance du 7 sept. 1790.

- Réflexions sur les corvées, 1775, BI, Ms 857, f. 216-247; Ms 865, f. 205.

- Révision des travaux de la première législature, 1792, BI, Ms 863, f. 201-205.

- $\quad$ [Sur l'affaire du juge de paix Larivière], 1792, BI, Ms 863, f. 289-292.

- Sur le décret du 19 juin 1790, BI, Ms 860, f. 270-274.

- [Tableau historique des progrès de l'esprit humain de 1793-1794], TH, p. 471-1116.

- $\quad$ [Textes isolés d'un tableauhistorique des progrès de l'esprit humain - 1770-1780] ; TH, p. $183-221$ 
- $\quad$ Traité ducalcul intégral,[1778-1782], BI, Ms 879, f. 1-322.

- $\quad$ Vie de Voltaire, 1789, BI, Ms 855, f. 482-631.

\section{$\underline{\text { Correspondance }}$}

- Condorcet à Anisson-Duperron, 20 nov. [1776], Saint-Pétersbourg, Archives de l'Institut d'histoire, K 11, pièce 52.

- Condorcet à Blagden, [mai-juin 1784], Londres, Royal Society, CB/ 1/ 3/ D15.

- Condorcet à Brissot, [31 déc. 1789], AN, 446 AP/7, pièce 78.

- Condorcet à Brissot, [26 fév. 1791], AN, 446 AP/7, pièce 74.

- Condorcet à Brissot, [oct. 1791], Paris, Musée des lettres et manuscrits, pièce $\mathrm{n}^{\circ} 43826$.

- Condorcet à Euler, 14 fév. [1784], Saint-Pétersbourg,Archives de l'Académie des sciences, F. 1, op. 3, n ${ }^{\circ} 68,1.48$.

- Condorcet à Fabroni, 14 fév. [1776], Pise,BibliotecaUniversitaria, BI, Ms 422.26/45.

- Condorcet à Frédéric II, [mai 1784], Berlin, Archives d'État, Rep. 96, Nr. 204 B.

- Condorcet à Frédéric II, 10 juil. 1786, Paris, Musée des lettres et manuscrits, pièce $\mathrm{n}^{\circ} 37883[=5]$.

- Condorcet à Hebert, [1774], Collection privée Jean-Daniel Candaux.

- Condorcet à Linné fils, 15 août 1778, Londres, Linnean Society, LinneanCorrespondence, vol. 3, f. 89-90.

- Condorcet à Saluces, [19 août 1784], Turin, Accademiadellescienze, carton 32225.

- Condorcet à Stanhope, 19 nov. 1776, U1590, C 13/13, Maidstone, Center for KentishStudies.

- Condorcet à Stanislas II Poniatowski, [17 avr. 1791 ?], Cracovie, BibliotekaJagiellonska, AutographenSammlung, Dig. ORP 000492.

- Condorcet à de Sivry, 21 août 1783, Saint-Pétersbourg, Archives de l'Institut d'histoire, K 11 , pièce 49 .

- Condorcet à A. Suard, [juin-juil. 1780], BnF, NAF 23639, f. 24-25.

- Condorcet à J.-B. Suard, [fin mai 1782], BnF,NAF 23639, f. 35-36.

- Condorcet à Thunberg, 5 sept. 1787, Saint-Pétersbourg, Bibliothèque nationale de Russie, F. 993 - op. 2, carton $36, \mathrm{n}^{\circ} 1374$.

- Condorcet à Trudaine, [mars 1775], AN, F 14/610.

- Condorcet à Trudaine, 16 nov. 1775, AN, F 14/610, dans E. Szulman(2013).

- Condorcet à Turgot, 4 déc. [1773], BI, Ms 7442, f. 34-35.

- Condorcet à Turgot, [10 mai 1774], collection privée-vente de Proyart, 60/35.

- Condorcet à Turgot, [fin mai 1774], BI, Ms 7442, f. 44-45.

- Condorcet à Turgot, [début mars 1775], Collection privée-vente de Proyart, 68/35.

- Condorcet à Turgot, [début de l'été 1776], Collection privée-vente de Proyart, 58/35.

\section{$\underline{\text { Autres documents }}$}

- [Certification de conformité d'un Extrait des registres de l'Académie royale des sciences du 29 août 1789], 2 sept. 1789, AAS, Pochette de séance du 29 août 1789.

- [Certification de conformité d'un Extrait des registres de l'Académie royale des sciences du 27 janvier 1790], 29 janv. 1790, AAS, Pochette de séance du 27 janv. 1790.

- [Certification de conformité d'un Extrait des registres de l'Académie royale des sciences du 7 août 1790], 21 août 1790, AAS, Pochette de séance du 4 août 1790.

- $\quad$ [Signature de Condorcet à cinq ans : Parrainage de Marie-Jean-Antoine Delattre], 10 août 1749, AD Aisne, 5 MI 1520 ; dans N. Rieucau (2009, p. 163). 


\section{Imprimés}

Correspondance

- Condorcet à A. Suard, [début août 1785], EB, p. 222-223.

- Condorcet à $M^{* * *}$ [La Méthérie], [juin 1790], OC, t. I, p. 327-330.

- Condorcet à Turgot, 10 oct. 1775 [date allographe ?], CH, p. 252-254.

- Condorcet à Turgot, 15 oct. [1775], CH, p. 258-259.

- J. de Lespinasse à Condorcet, 13 [juil. 1769], JNP, p. 29-30.

- J. de Lespinasse à Condorcet, 17 oct. 1775, JNP, p. 124-125.

- Lettre de M. de Condorcet à M. le comte Mathieu de Montmorency, député du bailliage de Monfort-l'Amaury, 30 août 1789, s. 1. n. d.

\section{AUTRES RÉFÉRENCES}

\section{Manuscrits}

- Armoiries de quelques familles de Ribemont, s. d., AD Aisne, Fonds Amédée Piette, 42 fi 68.

- CARdot Étienne, 13 thermidor an II [31 juil. 1794], Aux Citoyens Représentans du peuple composant le Comité de Sûreté Générale, AN, F7 4633, dos. 3.

- HozIERCharles-René d' (1697-1709), Armorial général de France, dressé en vertu de l'édit de novembre 1696, BnF, Fr. 32228-32262.

- Plumitif DES SÉANCES DE L'ACADÉMIE DES SCIENCES, années 1761, 1775, 1784 et 1787, AAS.

- PROCÉS-VERBAUX DES SÉANCES DE L'ACADÉMIE DES SCIENCES, année 1775, AAS.

- Vernet Madame, [1825 ?], Madame Vernet à Éliza O’Connor, Ms 848, f. 61-62.

\section{$\underline{\text { Imprimés }}$}

- BADINTER Élisabeth (2008), «Auguste de Keralio : un auxiliaire invisible de la République des sciences », Dix-huitième siècle, $\mathrm{n}^{\circ} 40$, p. 53-67.

- BRU Bernard \& CRÉPEL Pierre (1994), Condorcet - Arithmétique politique - Textes rares ou inédits (1767-1789), Paris : Ined.

- BRUNOT Ferdinand (1966), Histoire de la langue française des origines à nos jours, par Alexis François, t. VI (XVIII ${ }^{\mathrm{e}}$ siècle), $2^{\mathrm{e}}$ partie, Armand Colin.

- Bustarret Claire (1996), «Les instruments d'écriture, de l'indice au symbole », Genesis, $\mathrm{n}^{\circ} 10$, p. 175-191.

- Bustarret Claire (2004), « Analyse codicologique », TH, p. 1201-1223.

- Bustarret Claire (2009), "Approche codicologique du manuscrit moderne », dans Olga Anokhina \& Sabine Pétillon (dir.), Critique génétique. Concepts, méthodes, outils, SaintGermain-la-Blanche-Herbe, IMEC, coll. « Inventaires », p. 49-59.

- Bustarret Claire (2012), «Usages des supports d'écriture au XVIII ${ }^{\mathrm{e}}$ siècle : une esquisse codicologique », Genesis, n³4, p. 37-65.

- CAHEN Léon (1904), « Note sur les manuscrits de Condorcet conservés à la Bibliothèque de l'Institut », Séances et travaux de l'Académie des sciences morales et politiques - Comptes rendus, $\mathrm{n}^{\circ} 61, \mathrm{p} .779-785$.

- Chouillet Anne-Marie (1997), « Présentation », dans Anne-Marie Chouillet et Pierre Crépel (dir.), p. 15-17.

- Chouillet Anne-Marie \& CRÉPEl Pierre (dir.) (1997), Condorcet - Homme des Lumières et de la Révolution, Fontenay/Saint-Cloud : ENS Éditions. 
- CRÉPEl Pierre (1996), «Esquisse d'une histoire du Tableau historique », Mélanges de l'école française de Rome, Rome, École française de Rome, t. 108, p. 469-504.

- CRÉPEl Pierre \& Rieucau Nicolas (2005), «Condorcet's Social Mathematic - A Few Tables ", Social Choice and Welfare, vol. 25, n²-3, p. 243-285.

- GAUdRIAUlt Raymond (1995), Filigranes et autres caractéristiques des papiers fabriqués en France aux XVII e et XVIII ${ }^{e}$ siècles, Paris : CNRS Editions.

- HAY Louis (1993), "Les manuscrits au laboratoire », dans Louis Hay (dir.), Les manuscrits des écrivains, [Paris :] CNRS Editions/Hachette, p. 122-137.

- LAfFitTe-LaRnaudie Anne-Marie (1997), « Le secrétaire de Condorcet : Étienne Cardot (17541847) », dans Anne-Marie Chouillet \& Pierre Crépel (dir.), p. 37-53.

- L'Eleu André (1913), «Le Parrain du Cousin Jacques : Louis Fromage de Longueville (17321786) », Bulletin de la Société académique de Laon, St-Quentin : D. Antoine, t. XXXV, p. 27300.

- Moureau François (1999), «Fromage de Longueville, Louis (1732 ?-1786)», dans Jean Sgard (dir.), Dictionnaire des journalistes 1600-1789, Oxford: Voltaire Foundation, vol. II, p. 419-420.

- RĖGlemENT ORDONNÉ PAR LE ROI POUR L'ACAdÉMIE ROYALE DES SCIENCES (26 janv. 1699), dans Éric Brian \& Christiane Demeulenaere-Douyère (dir.), Histoire et mémoire de l'Académie des sciences, Londres, Paris, New York : Éditions TEC \& DOC, 1996, p. 409-413.

- RIEUCAU Nicolas (2003), « La neige avait-elle recouvert le volcan ? L'écriture par Condorcet du Tableau historique des progrès de l'esprit humain », Genesis, n²2, p. 12-35.

- RIEUCAU Nicolas (2004 a), «Introduction [aux Textes isolés] », TH, p. 175-183.

- RIEUCAU Nicolas (2004 b), « Descriptif matériel », TH, p. 1137-1199.

- RIEUCAU Nicolas (2009), «L'économie d'un savant : l'œuvre de Condorcet, des archives à l'interprétation », Synthèse pour l'habilitation à diriger des recherches, Université Paris 8.

- Rousseau Jean-Jacques (1770), «Les confessions», J. J. Rousseau. Euvres complètes, Gallimard, Bibliothèque de la Pléiade, 1959, t. I, p. 1-656.

- SzULMAN ÉRIC (2013), « Aux sources d'une activité administrative : la correspondance entre Condorcet et Trudaine au sujet de la navigation intérieure sous le ministère Turgot », $L a$ correspondance de Condorcet: documents inédits, nouveaux éclairages, Ferney-Voltaire : Centre international d'étude du XVIII ${ }^{\mathrm{e}}$ siècle (à paraître).

- TARELlo Giovanni (1975), «Idee e atteggiamenti sulla repressione penale », Materiali per unastoria della cultura giuridica, Bologna : Societàeditrice il Mulino, vol. V, p. 353-451.

- Tremblot Jean (1929-1931), « Bibliothèques et armoiries des académies royales de Paris », Bulletin du bibliophile et du bibliothécaire, 1929 : p. 390-399, 1930 : p. 270-283, 1931 : p. 121-133.

- VinOt Bernard (1989 a), «Condorcet et sa terre picarde », dans Pierre Crépel et Christian Gilain (dir.), Condorcet mathématicien, économiste, philosophe, homme politique, [Paris :] Minerve, p. 416-424.

- VinOT Bernard (1989 b), «Condorcet et sa terre natale », Annales Historiques de la Révolution Française, n ${ }^{\circ}$ 275, janv.-mars, p. 1-15.

\section{Pages ou sites internet}

- CALENDRIER DES PRESENCES ACADEMIQUES DE CONDORCET : www.inventairecondorcet.com/fra/page/11

- INVENTAIRE CONDORCET : www.inventaire-condorcet.com

- Kölving Ulla, [Présentation de] Voltaire, Le Siècle de Louis XIV, l'édition de 1751 : http://c18.net/vo/vo_textes_siecle.php?div1 $=45$ 